\title{
Construction of Pentacyclic Limonoid Skeletons via Radical Cascade Reactions
}

Hiroyuki Mutoh, Shu Nakamura, Koichi Hagiwara, and Masayuki Inoue*

Graduate School of Pharmaceutical Sciences

The University of Tokyo, Hongo, Bunkyo-ku, Tokyo 113-0033, Japan

Fax: (+81)3-5841-0568

E-mail: inoue@mol.f.u-tokyo.ac.jp

Supplementary Material

29 pages

Contents:

page

1. Determination of stereochemistries of compounds $13,14,15,4 \mathbf{a}, \mathbf{3 a}-\boldsymbol{\beta}, \mathbf{3 b}$ $\boldsymbol{\alpha}$, and $\mathbf{3 c - \alpha}$

2. NMR spectra of newly synthesized compounds 
1. Determination of stereochemistries of compounds $13,14,15,4 \mathrm{c}, 3 \mathrm{a}-\boldsymbol{\beta}, 3 \mathrm{~b}-\alpha$, and $3 \mathrm{c}-\alpha$ 1-1. Determination of the C6-stereochemistries of compounds 13 and 14.

The C6-stereochemistries of compounds $\mathbf{1 3}$ and $\mathbf{1 4}$ were determined by the ${ }^{1} \mathrm{H}$ NMR chemical shift of the C6-protons. Compound $\mathbf{1 4}$ has the more downshielded signal for C6-H $(\delta 6.20$ in $\mathrm{CDCl}_{3}$ ) due to the effect of the neighboring $\mathrm{Br}$ atom.

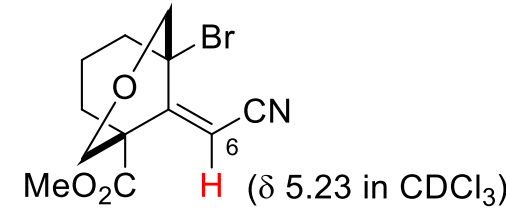

13

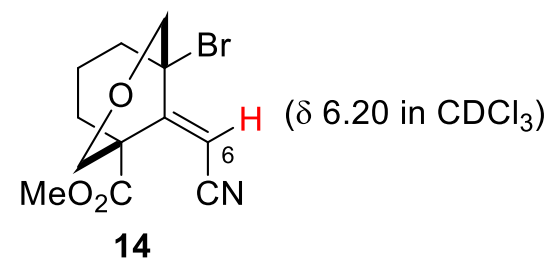

14

\section{1-2. Determination of the C5-stereochemistry of compound 15.}

The C5-stereochemistry of compound $\mathbf{1 5}$ was determined by the NOESY experiment of the derivatized 17- $\alpha$.

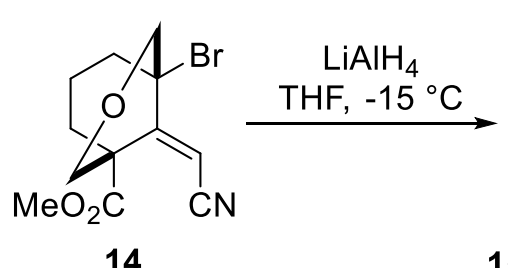

14

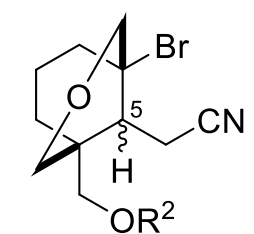

$15(\alpha-H: \beta-H=1: 1.6)$

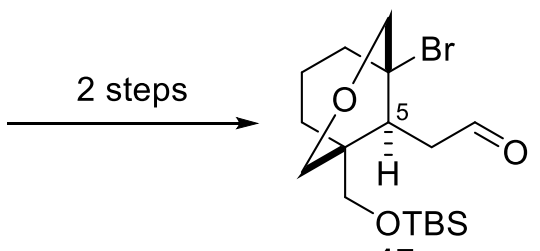

17- $\alpha$

$13 \%$ (3 steps)

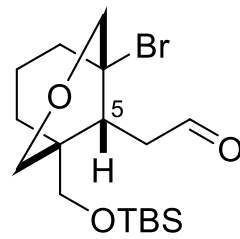

$17-\beta$

$21 \%$ (3 steps)

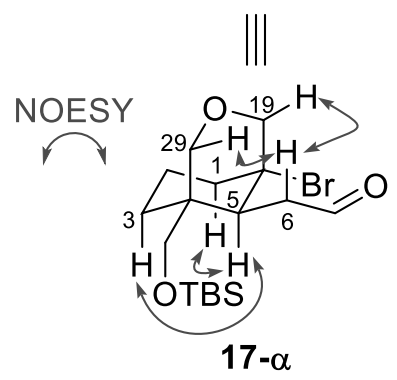

\section{1-3. Determination of the C8-stereochemistry of compound 4a.}

The C8-stereochemistry of compound $\mathbf{4 a}$ was determined by the NOE experiment of the derivatized $\mathbf{4 c}$.

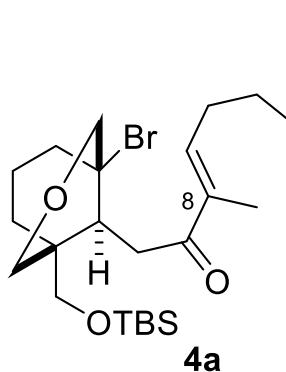

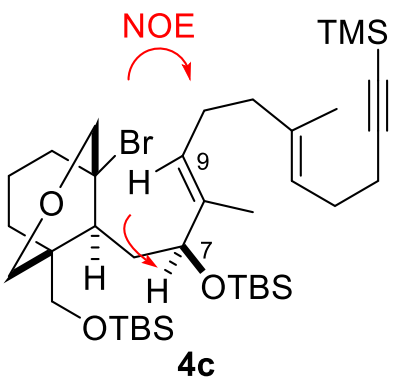


1-4. Determination of the stereochemistry of compound 3a- $\beta$.

The stereochemistry of compound $\mathbf{3 a -} \boldsymbol{\beta}$ was determined by the NOESY and NOE experiments.
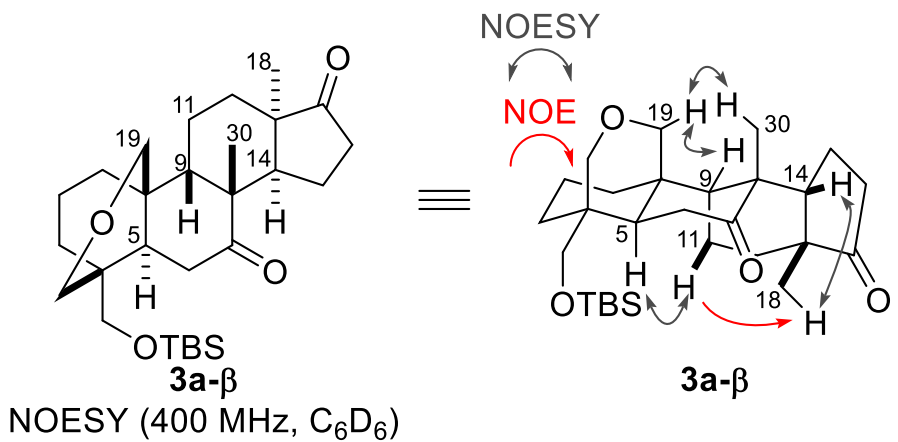

\section{1-5. Determination of the stereochemistry of compound $3 b-\alpha$.}

The stereochemistry of compound $\mathbf{3 b - \alpha}$ was determined by the NOESY experiment.
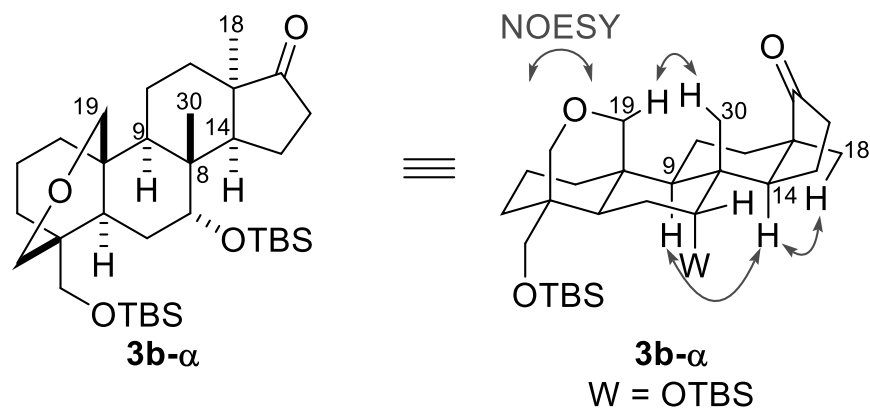

\section{1-6. Determination of the stereochemistry of compound 3c- $\alpha$.}

The stereochemistry of compound $\mathbf{3 c - \alpha}$ was determined by the NOESY experiment.
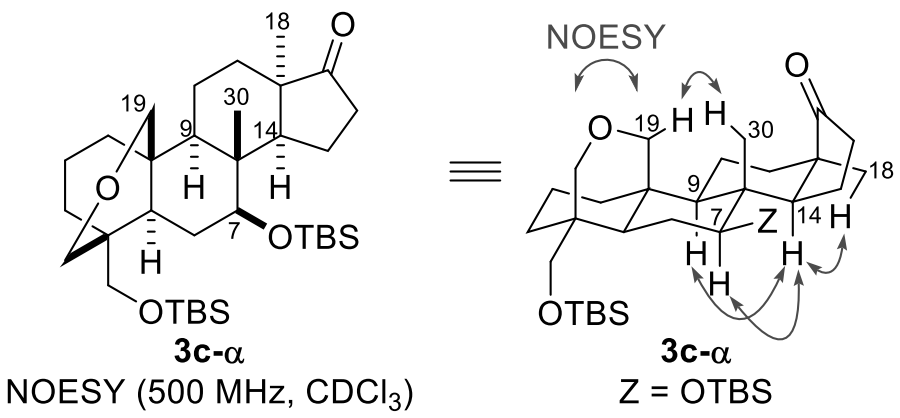
<smiles>OCC12CCCC(CO)(CC1)C2O</smiles>

10

${ }^{1} \mathrm{H}$ NMR $\left(400 \mathrm{MHz}, \mathrm{CD}_{3} \mathrm{OD}\right)$

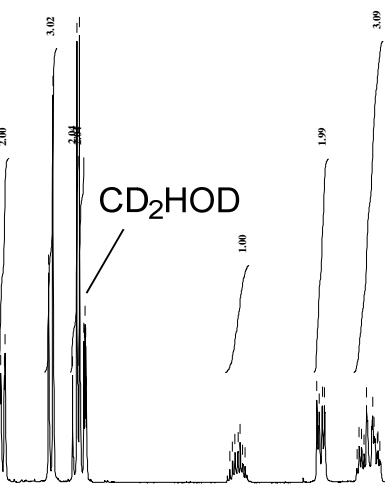

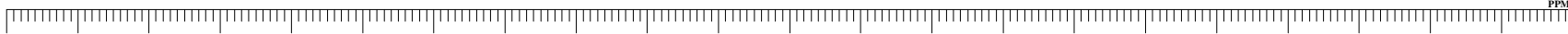

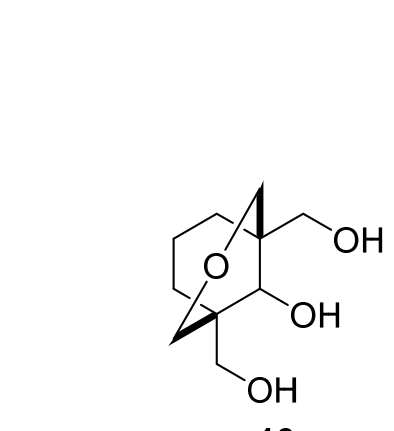

10

${ }^{13} \mathrm{C}\left\{{ }^{1} \mathrm{H}\right\} \operatorname{NMR}\left(100 \mathrm{MHz}, \mathrm{CD}_{3} \mathrm{OD}\right)$

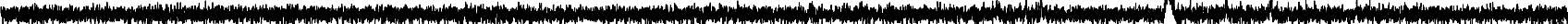

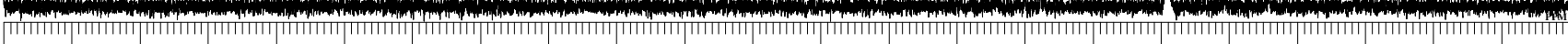

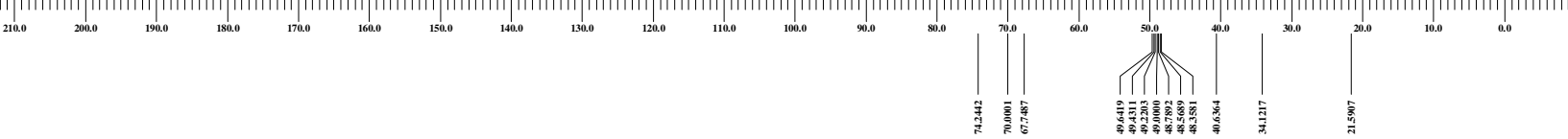




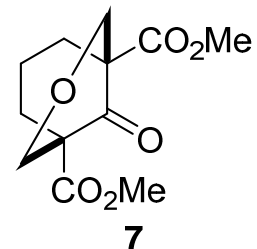

7

${ }^{1} \mathrm{H} \mathrm{NMR}\left(400 \mathrm{MHz}, \mathrm{CDCl}_{3}\right)$

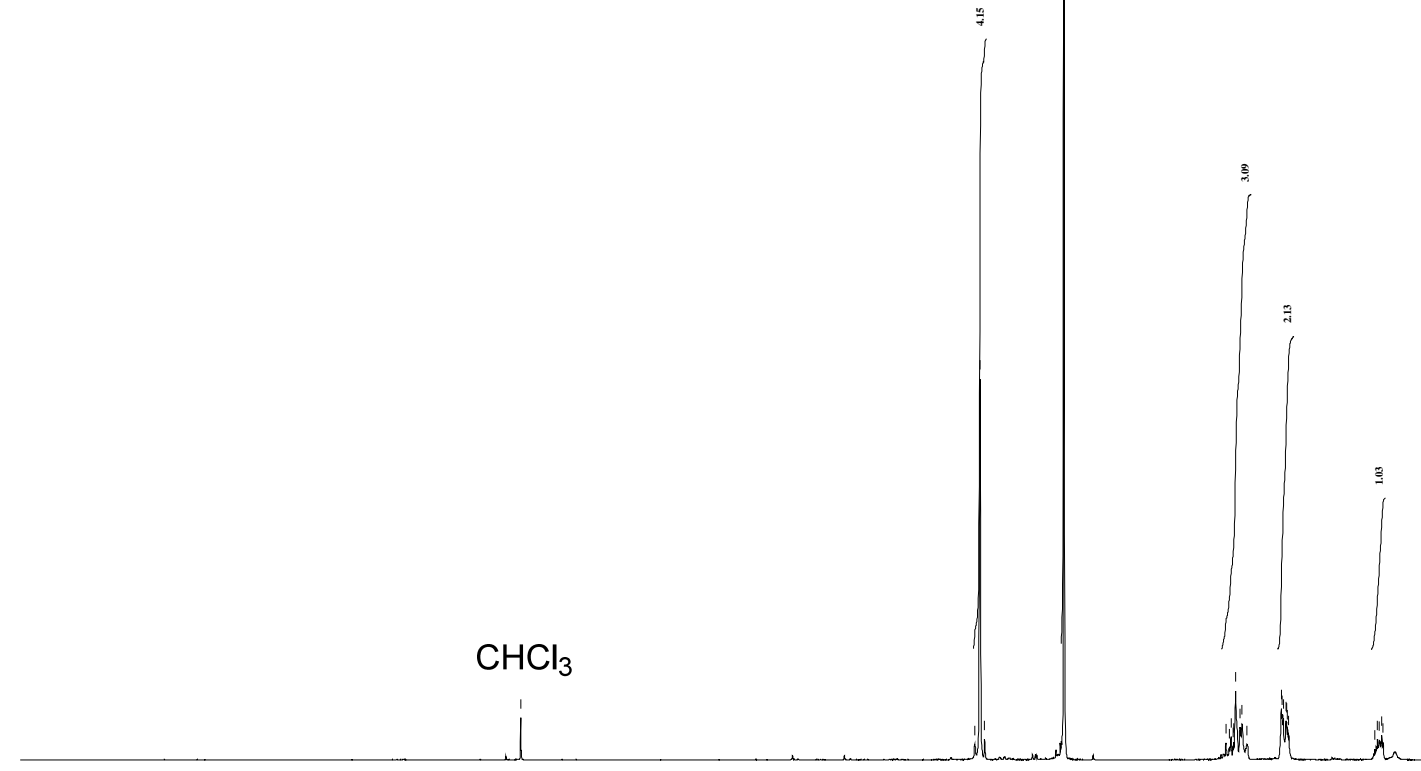

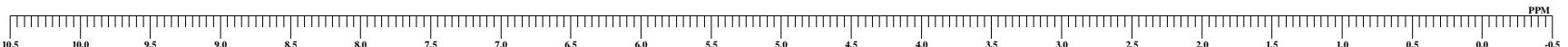

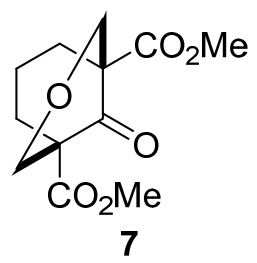

$\mathrm{CDCl}_{3}$

${ }^{13} \mathrm{C}\left\{{ }^{1} \mathrm{H}\right\}$ NMR $\left(100 \mathrm{MHz}, \mathrm{CDCl}_{3}\right)$

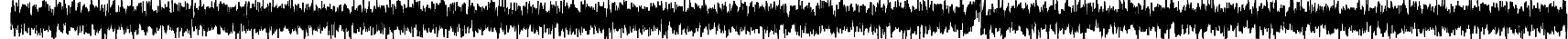

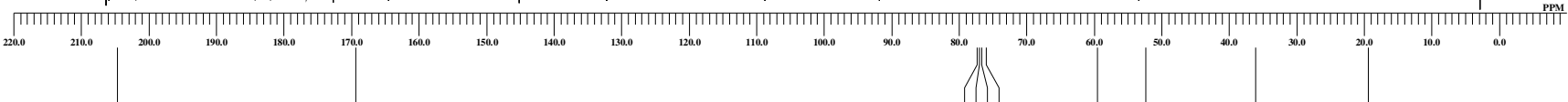


<smiles>COC(=O)C12CCCC(C)(CC1(Br)OC)C2=O</smiles>

12

${ }^{1} \mathrm{H} \mathrm{NMR}\left(400 \mathrm{MHz}, \mathrm{CDCl}_{3}\right)$
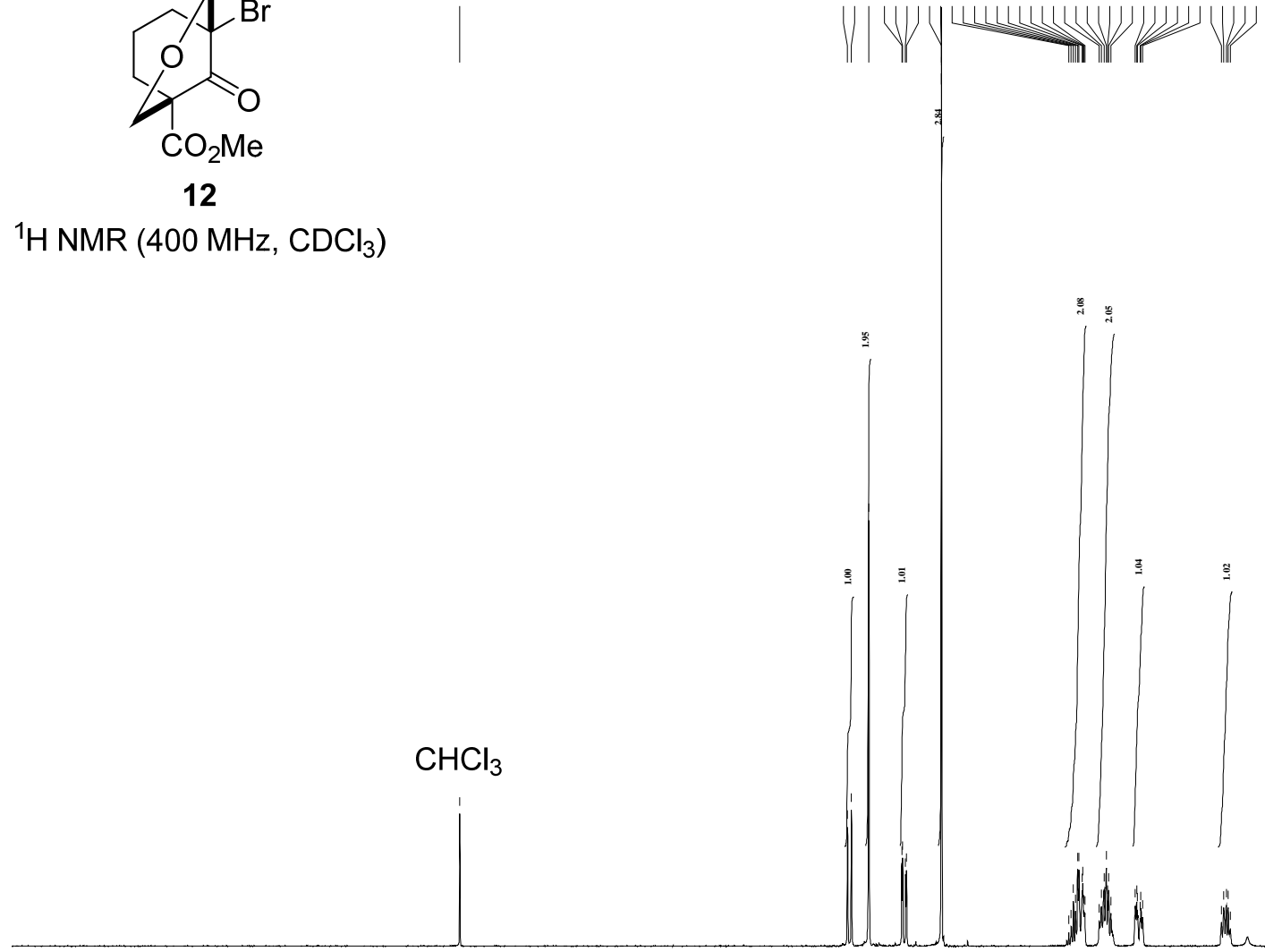

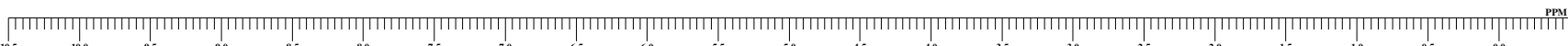

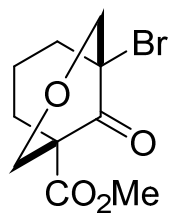

12

${ }^{13} \mathrm{C}\left\{{ }^{1} \mathrm{H}\right\} \mathrm{NMR}\left(100 \mathrm{MHz}, \mathrm{CDCl}_{3}\right)$

$\mathrm{CDCl}_{3}$

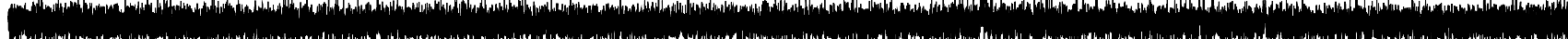

年 Tाता

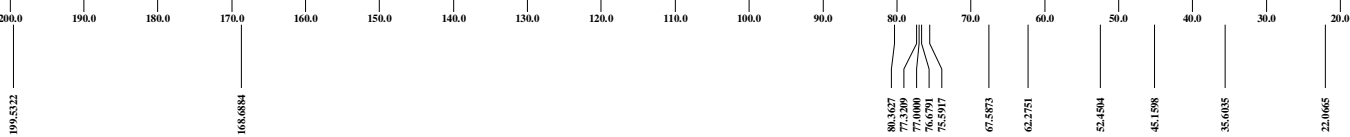




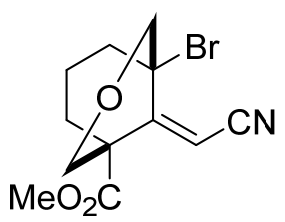

13

${ }^{1} \mathrm{H} \mathrm{NMR}\left(400 \mathrm{MHz}, \mathrm{CDCl}_{3}\right)$

\section{․․․}

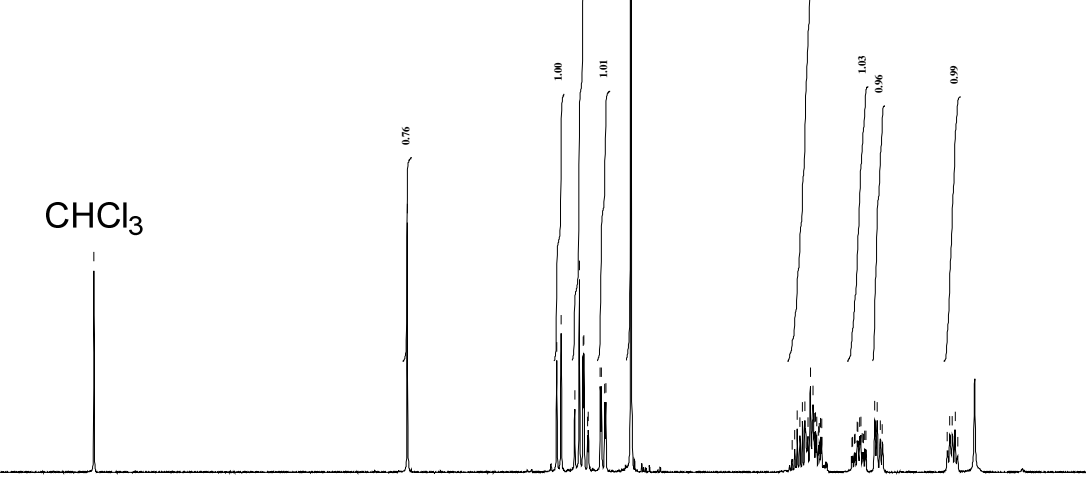

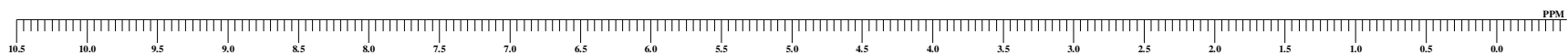

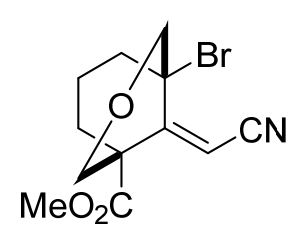

13

${ }^{13} \mathrm{C}\left\{{ }^{1} \mathrm{H}\right\} \mathrm{NMR}\left(100 \mathrm{MHz}, \mathrm{CDCl}_{3}\right)$

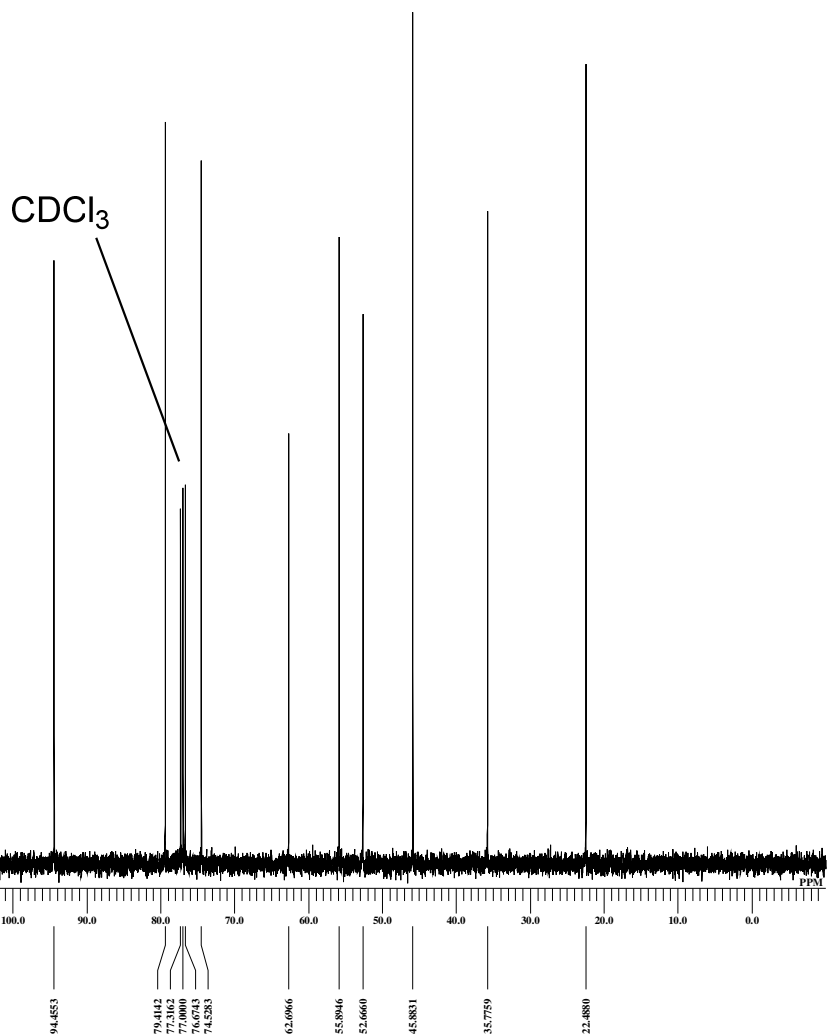




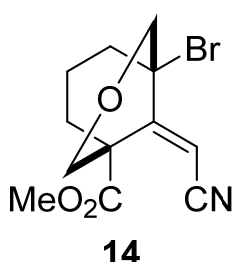

14

${ }^{1} \mathrm{HNMR}\left(400 \mathrm{MHz}, \mathrm{CDCl}_{3}\right)$

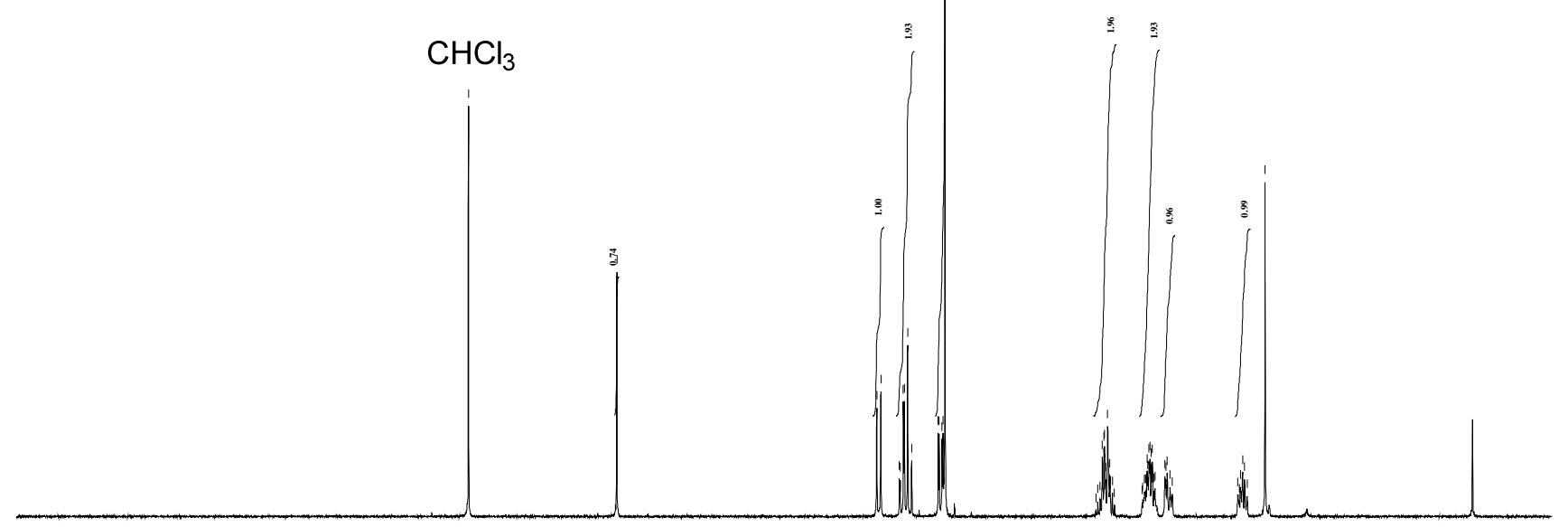

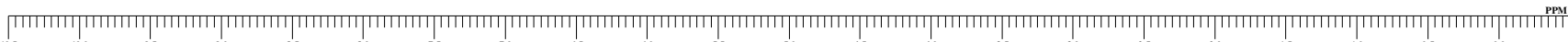

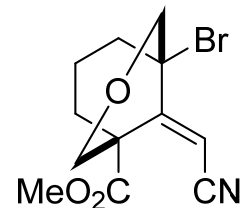

14

${ }^{13} \mathrm{C}\left\{{ }^{1} \mathrm{H}\right\} \mathrm{NMR}\left(100 \mathrm{MHz}, \mathrm{CDCl}_{3}\right)$

$\mathrm{CDCl}_{3}$

Whow

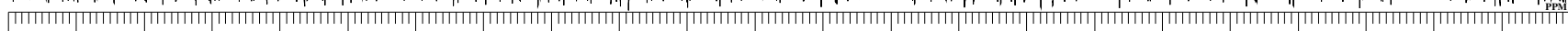

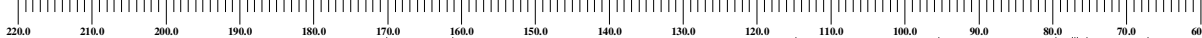

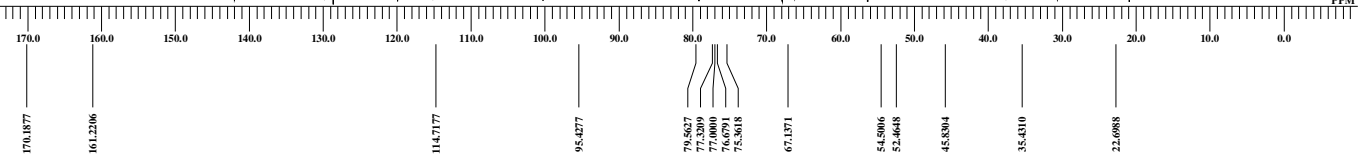




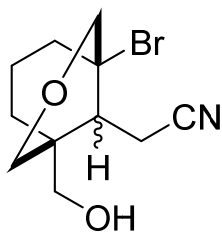

\section{$\mathrm{CHCl}_{3}$}

$15(\alpha-H: \beta-H=1: 1.6)$

${ }^{1} \mathrm{H}$ NMR $\left(400 \mathrm{MHz}, \mathrm{CDCl}_{3}\right)$

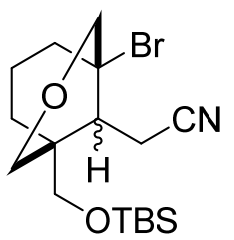

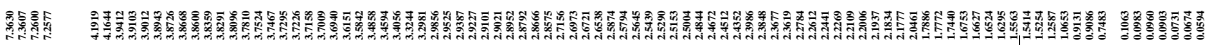

$16(\alpha-\mathrm{H}: \beta-\mathrm{H}=1: 1.4)$

${ }^{1} \mathrm{H}$ NMR $\left(400 \mathrm{MHz}, \mathrm{CDCl}_{3}\right)$

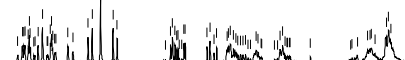

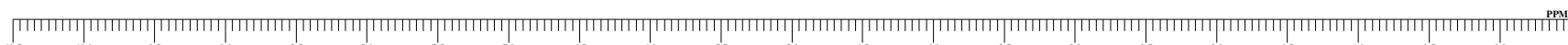


<smiles>C[Se+](C)(C)OCC12CCCC(Br)(CO1)C2CC=O</smiles>

17- $\alpha$

${ }^{1} \mathrm{H} \mathrm{NMR}\left(400 \mathrm{MHz}, \mathrm{CDCl}_{3}\right)$

$\mathrm{CHCl}_{3}$

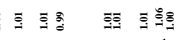

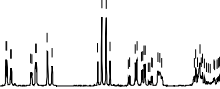

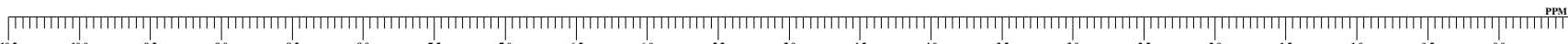<smiles>O=CCC1C2(Br)CCCC1(CO[Sb])CC2</smiles>

17- $\alpha$

${ }^{13} \mathrm{C}\left\{{ }^{1} \mathrm{H}\right\} \mathrm{NMR}\left(100 \mathrm{MHz}, \mathrm{CDCl}_{3}\right)$
$\mathrm{CDCl}_{3}$

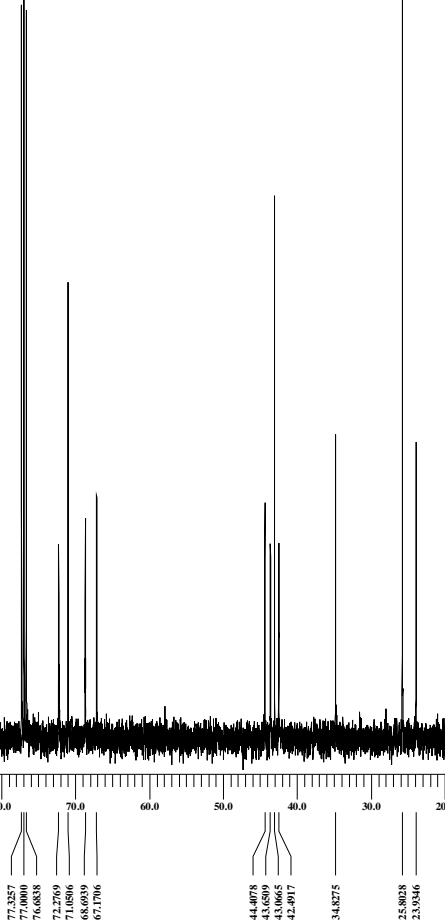

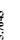



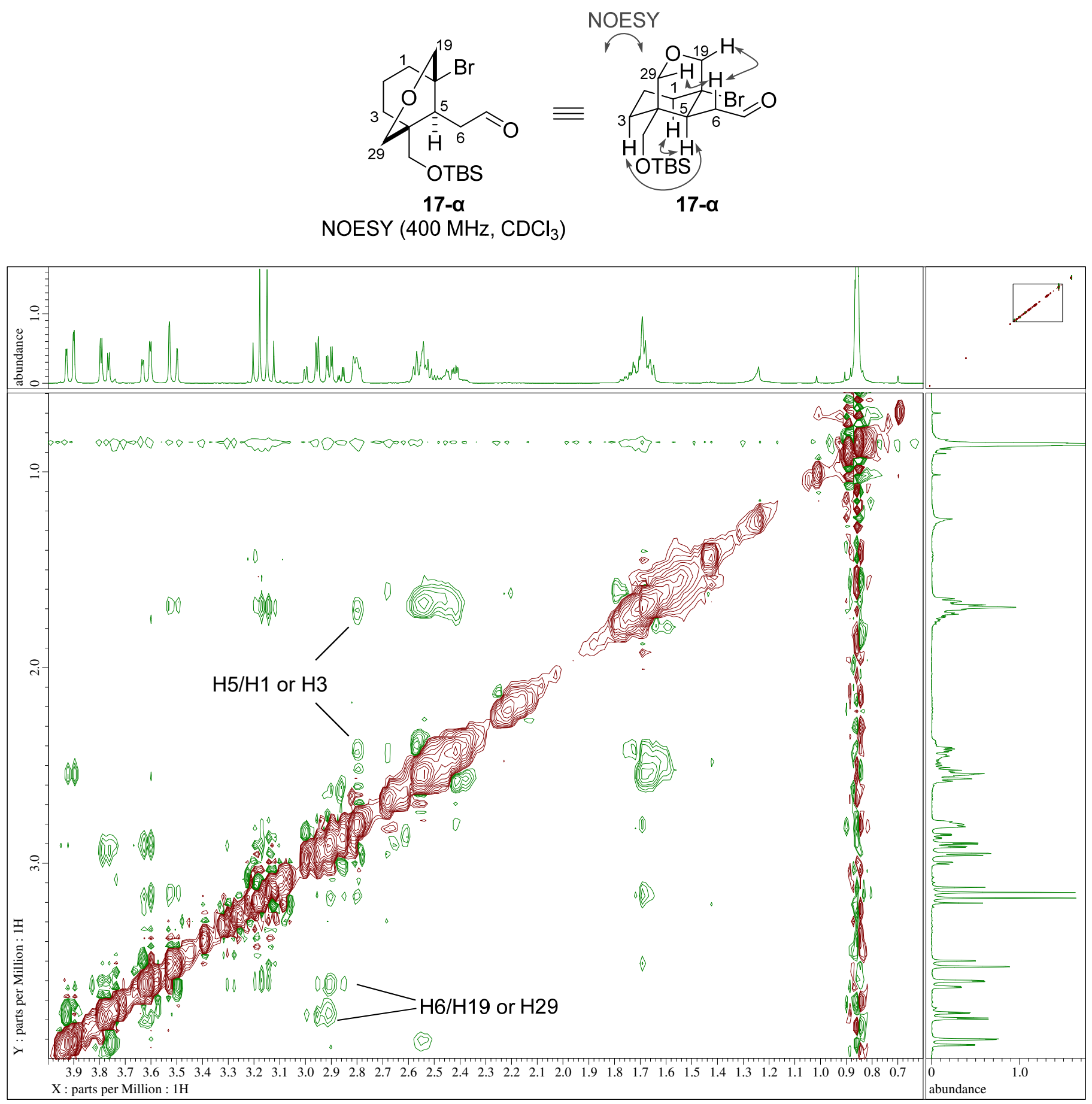
<smiles>O=CCC1(Br)C2(Br)CCCC1(CO[O+]=[Se])CC2</smiles>

$17-\beta$

${ }^{1} \mathrm{H} \mathrm{NMR}\left(400 \mathrm{MHz}, \mathrm{CDCl}_{3}\right)$

$\mathrm{CHCl}_{3}$

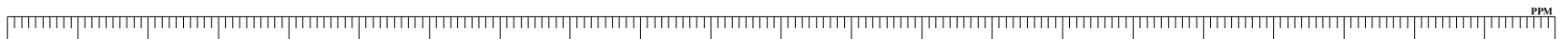
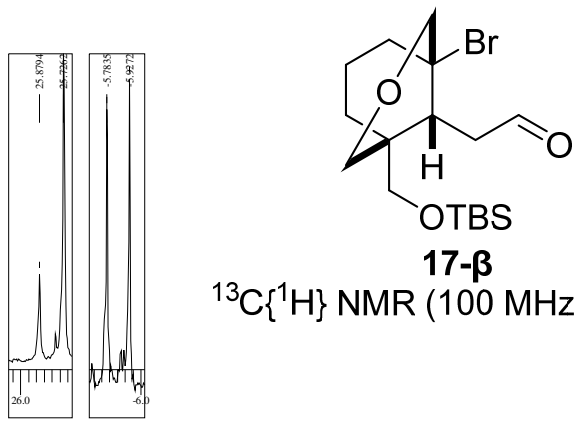

OTBS

17- $\beta$

${ }^{13} \mathrm{C}\left\{{ }^{1} \mathrm{H}\right\} \mathrm{NMR}\left(100 \mathrm{MHz}, \mathrm{CDCl}_{3}\right)$

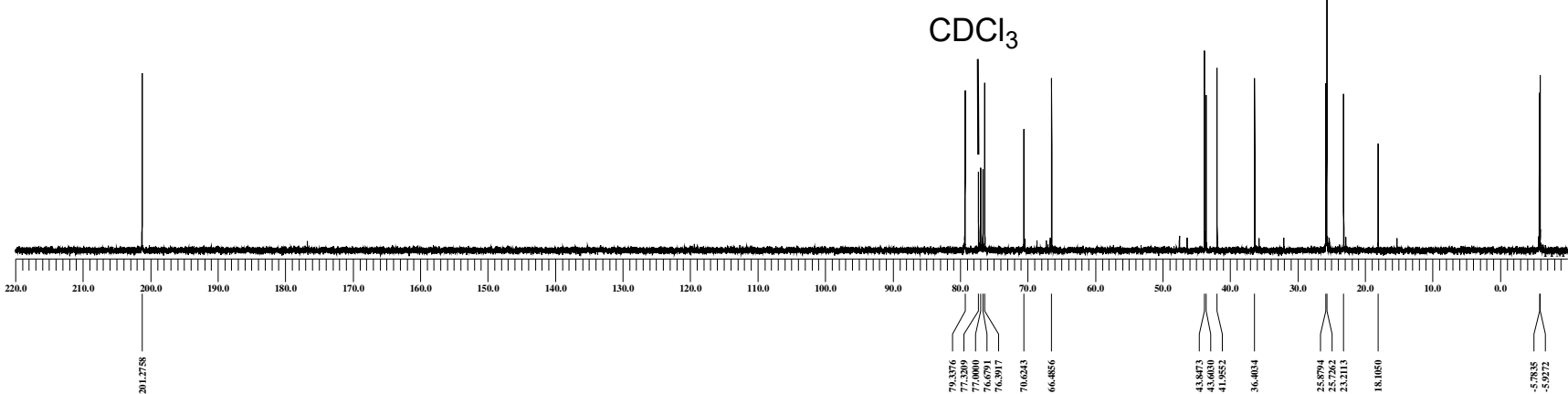




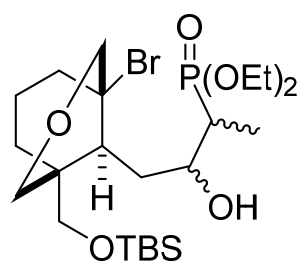

18

${ }^{1} \mathrm{H}$ NMR $\left(500 \mathrm{MHz}, \mathrm{CDCl}_{3}\right)$

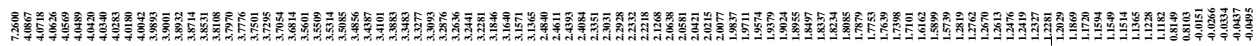

\section{$\mathrm{CHCl}_{3}$}

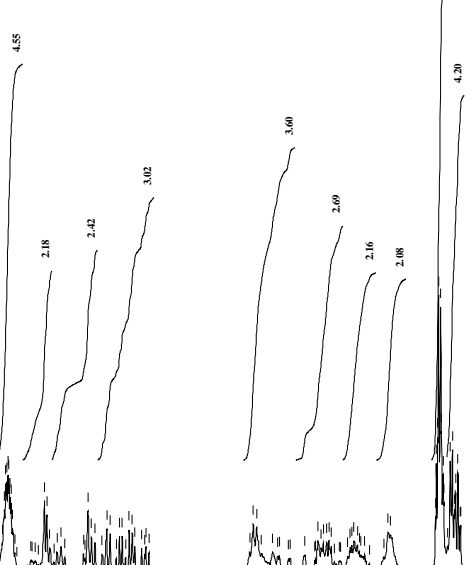

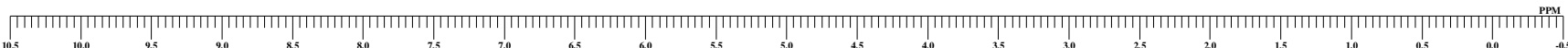
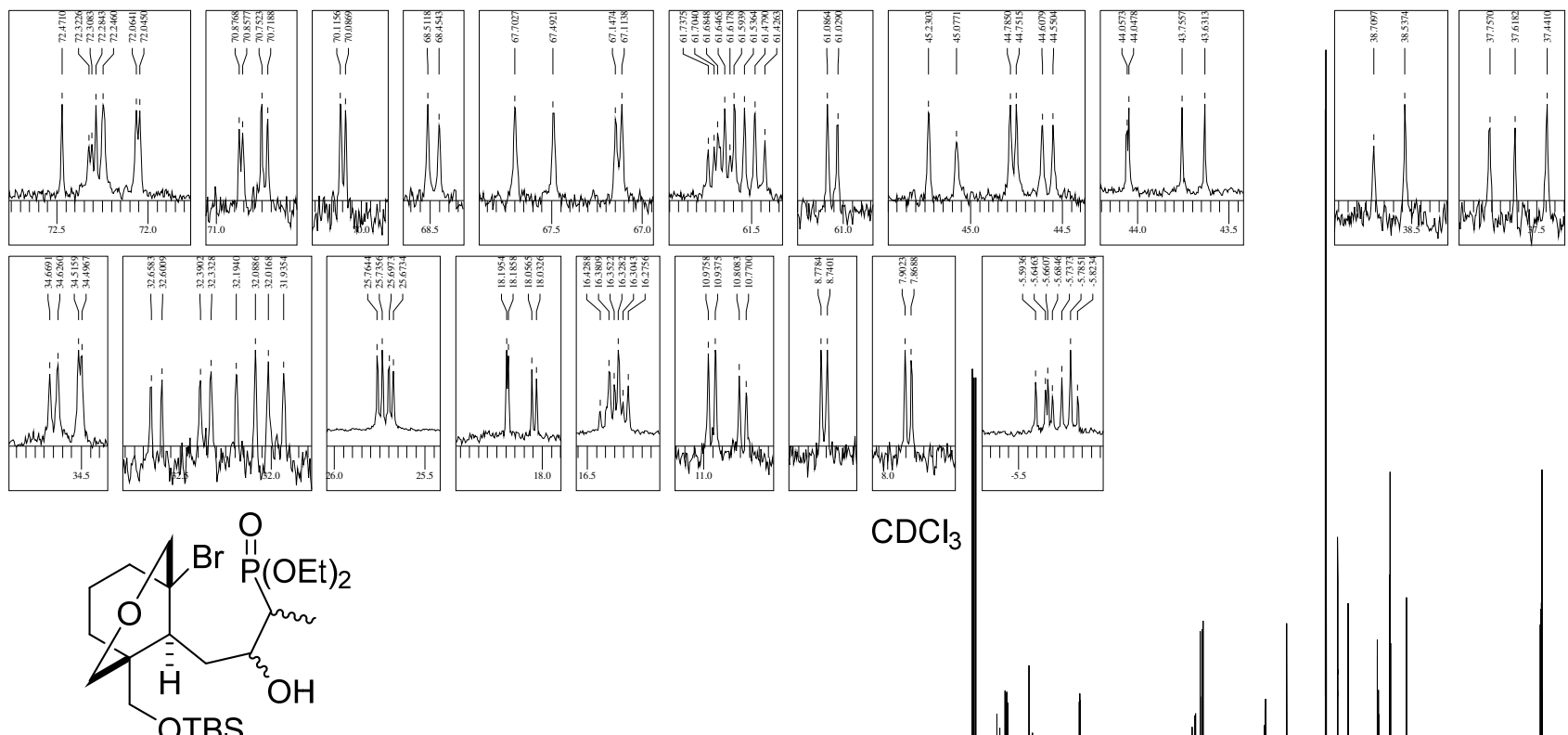

18

${ }^{13} \mathrm{C}\left\{{ }^{1} \mathrm{H}\right\} \operatorname{NMR}\left(125 \mathrm{MHz}, \mathrm{CDCl}_{3}\right)$

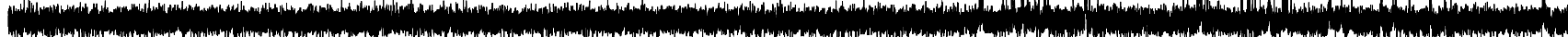

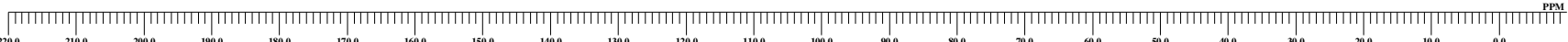

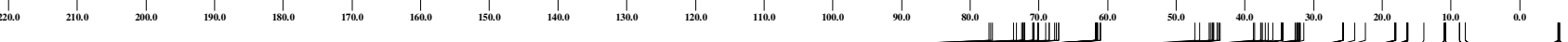

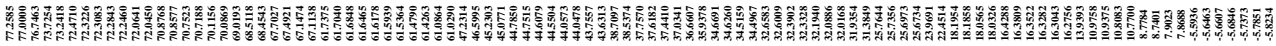




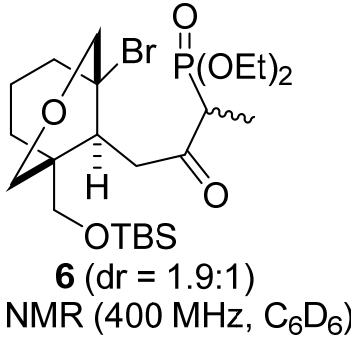

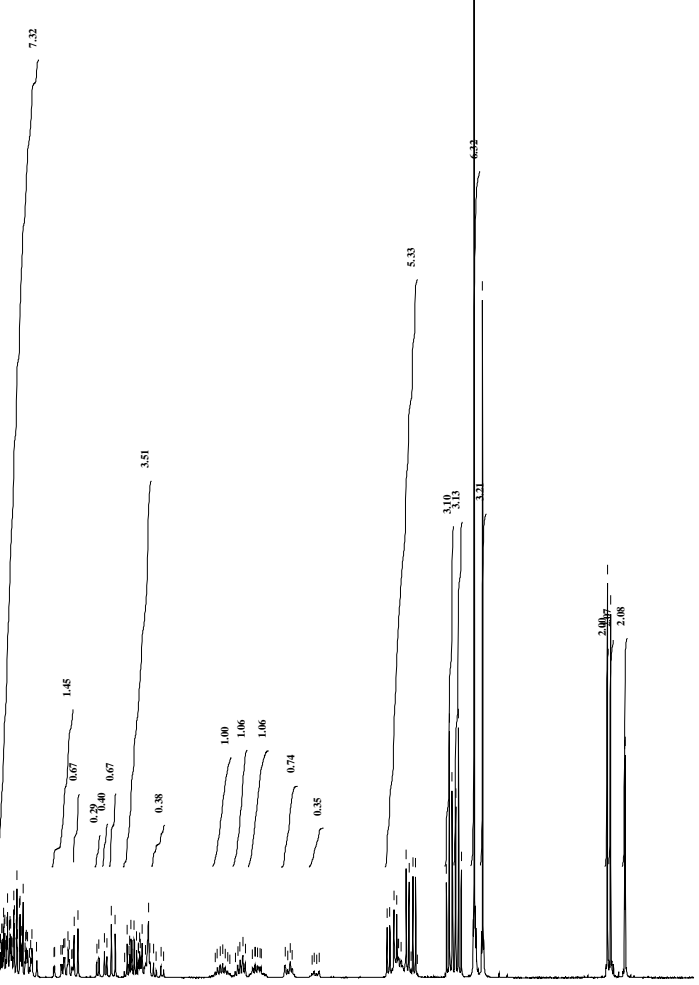

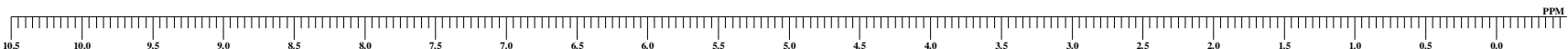
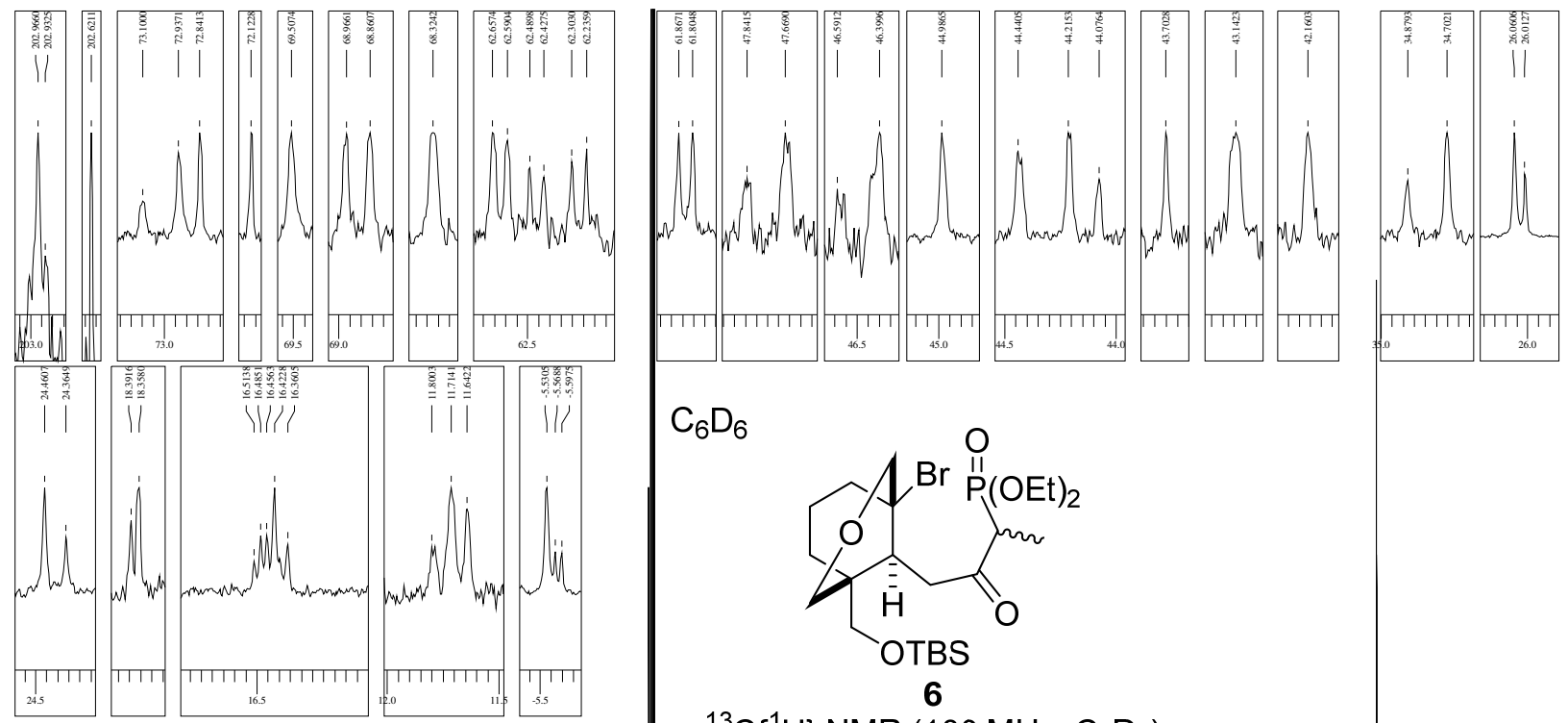

$\mathrm{C}_{6} \mathrm{D}_{6}$

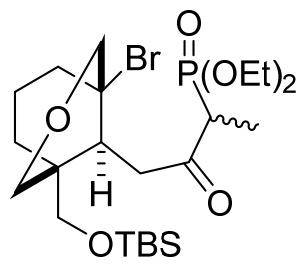

6

${ }^{13} \mathrm{C}\left\{{ }^{1} \mathrm{H}\right\} \mathrm{NMR}\left(100 \mathrm{MHz}, \mathrm{C}_{6} \mathrm{D}_{6}\right)$

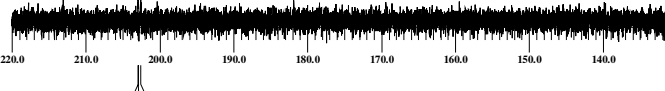

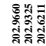




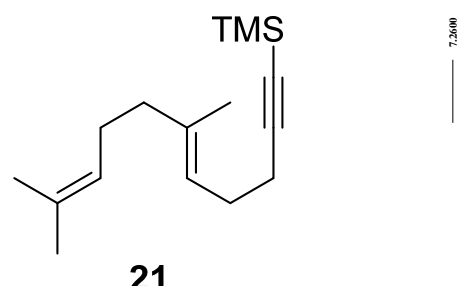

21

${ }^{1} \mathrm{H}$ NMR $\left(400 \mathrm{MHz}, \mathrm{CDCl}_{3}\right)$

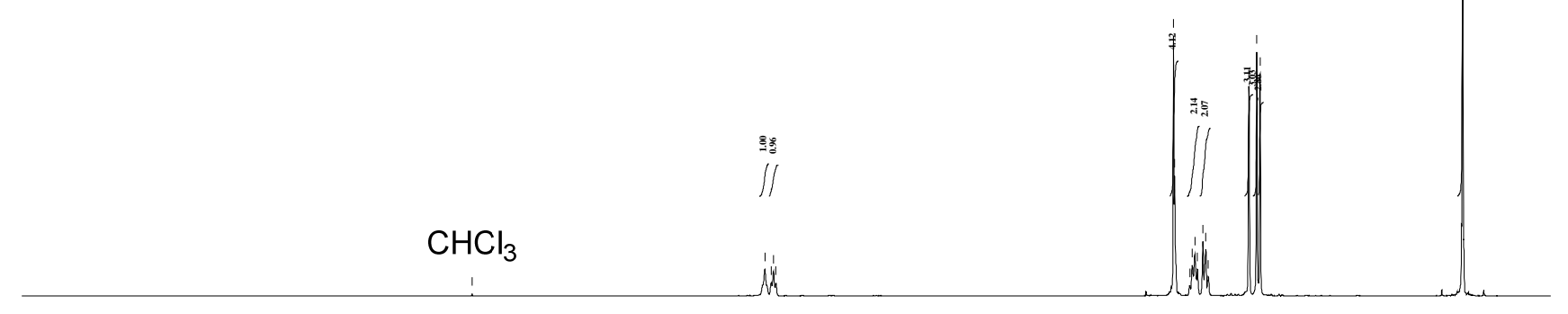

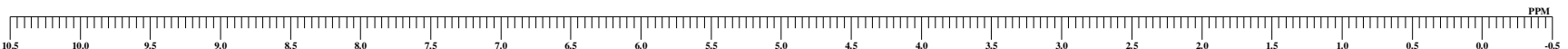

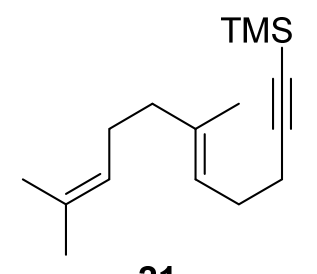

21

${ }^{13} \mathrm{C}\left\{{ }^{1} \mathrm{H}\right\} \operatorname{NMR}\left(100 \mathrm{MHz}, \mathrm{CDCl}_{3}\right)$

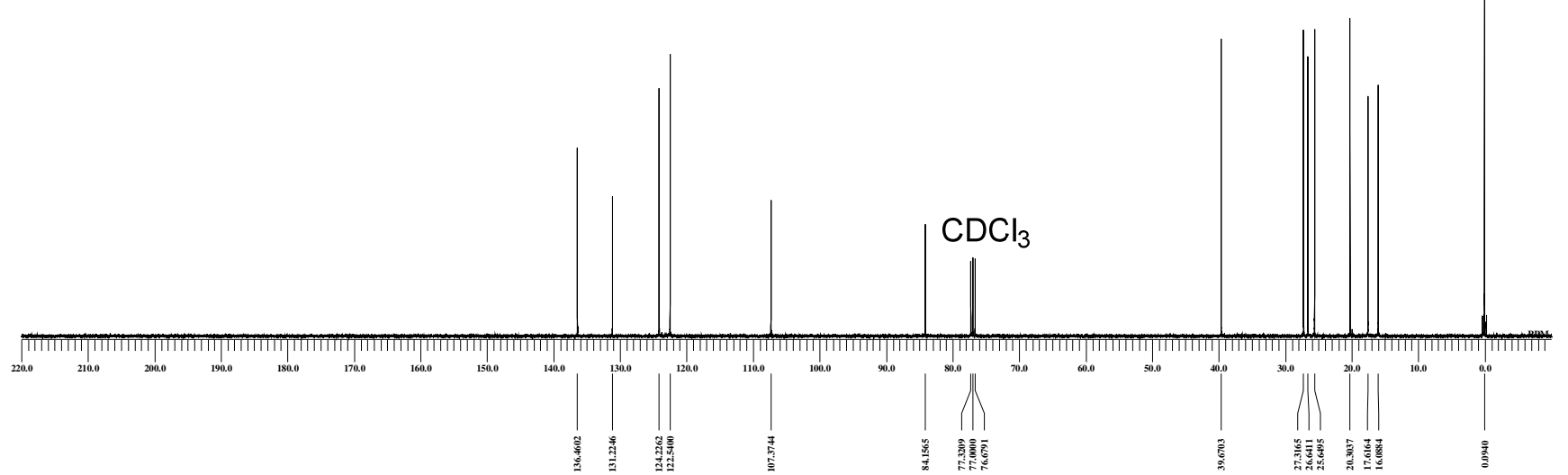


<smiles>CC#CCCCC(C)CCC(C)(C)O</smiles>

22

${ }^{1} \mathrm{H}$ NMR $\left(400 \mathrm{MHz}, \mathrm{CDCl}_{3}\right)$

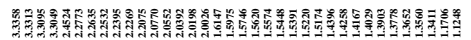

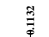

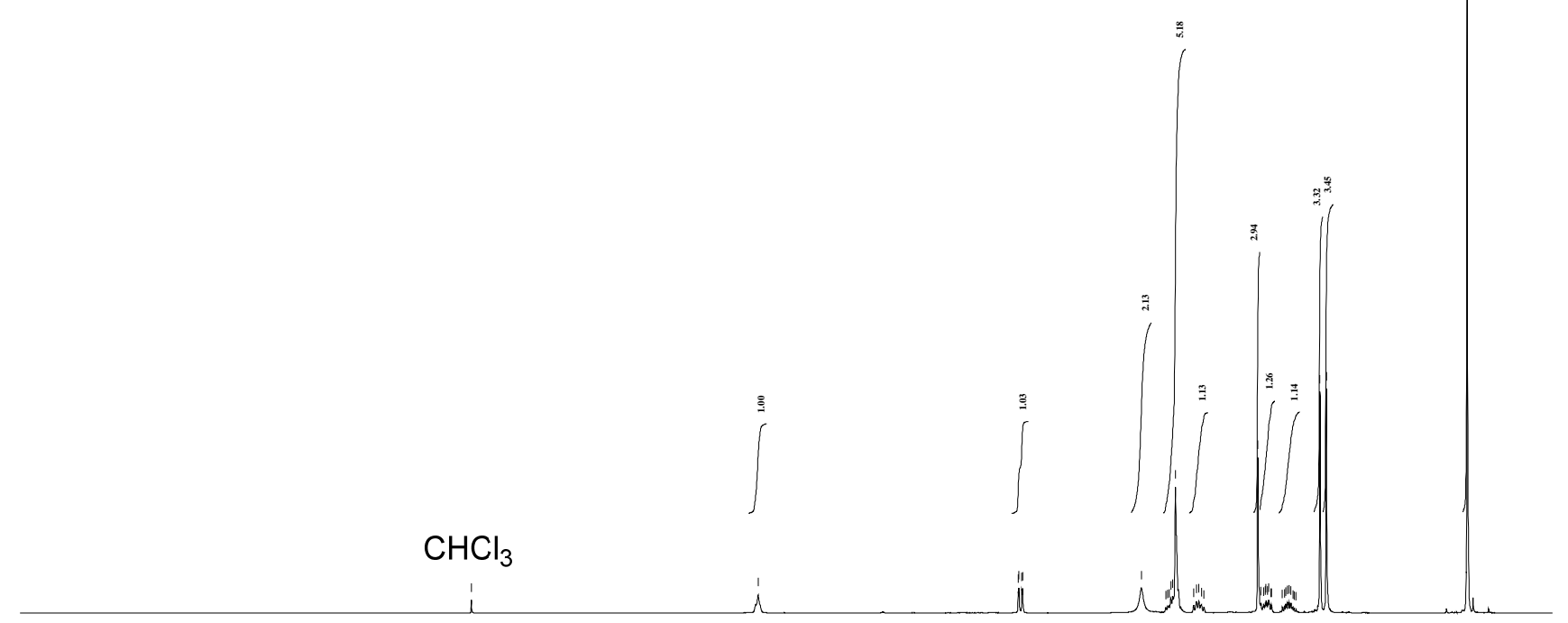

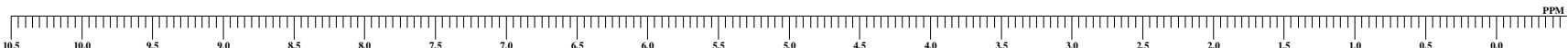<smiles>CC(C)=CCCC(O)C(C)(C)O</smiles>

22

${ }^{13} \mathrm{C}\left\{{ }^{1} \mathrm{H}\right\} \operatorname{NMR}\left(100 \mathrm{MHz}, \mathrm{CDCl}_{3}\right)$

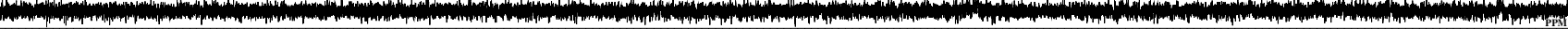

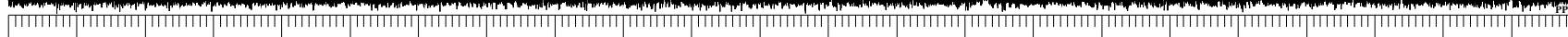<smiles>[GeH3]</smiles> 

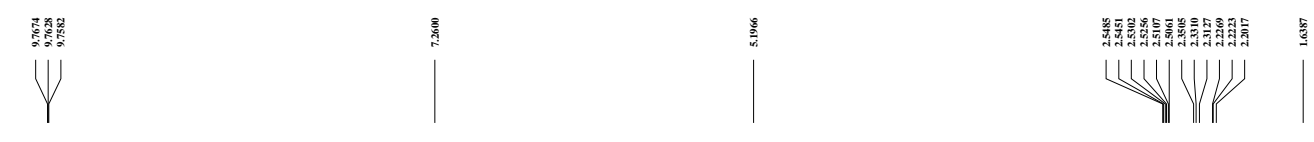<smiles>CC(C)=CCCC=C(C)CCC=O</smiles>

5

${ }^{1} \mathrm{H}$ NMR $\left(400 \mathrm{MHz}, \mathrm{CDCl}_{3}\right)$

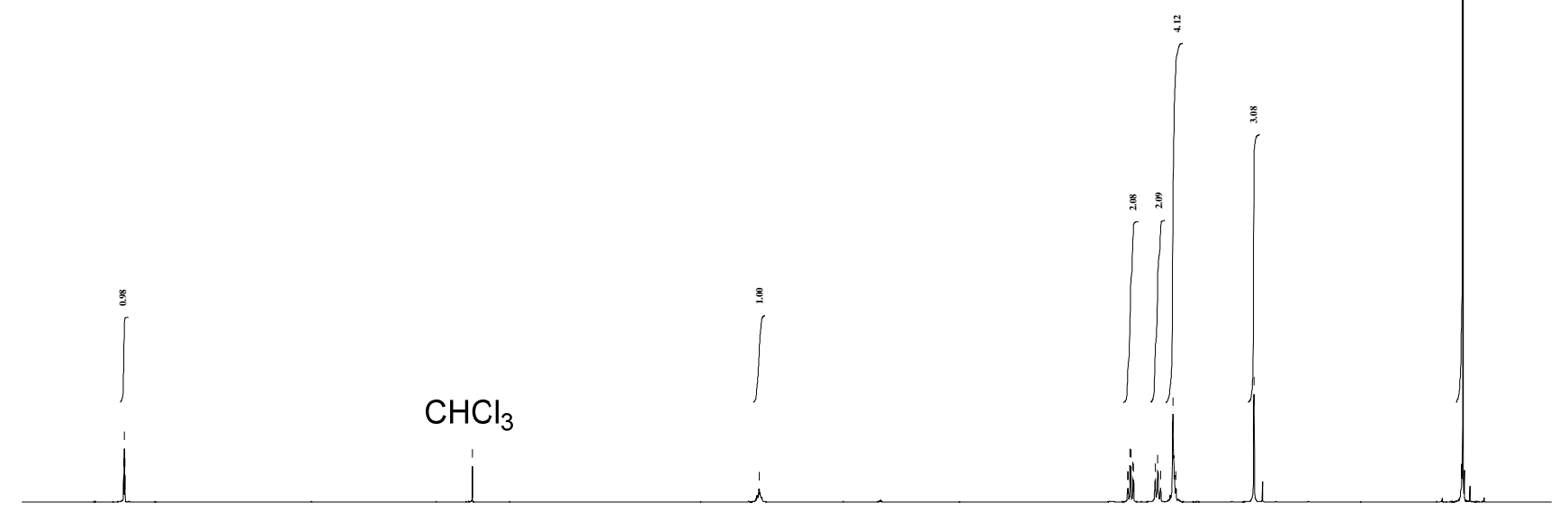

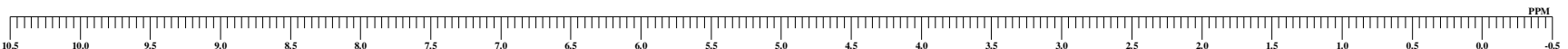

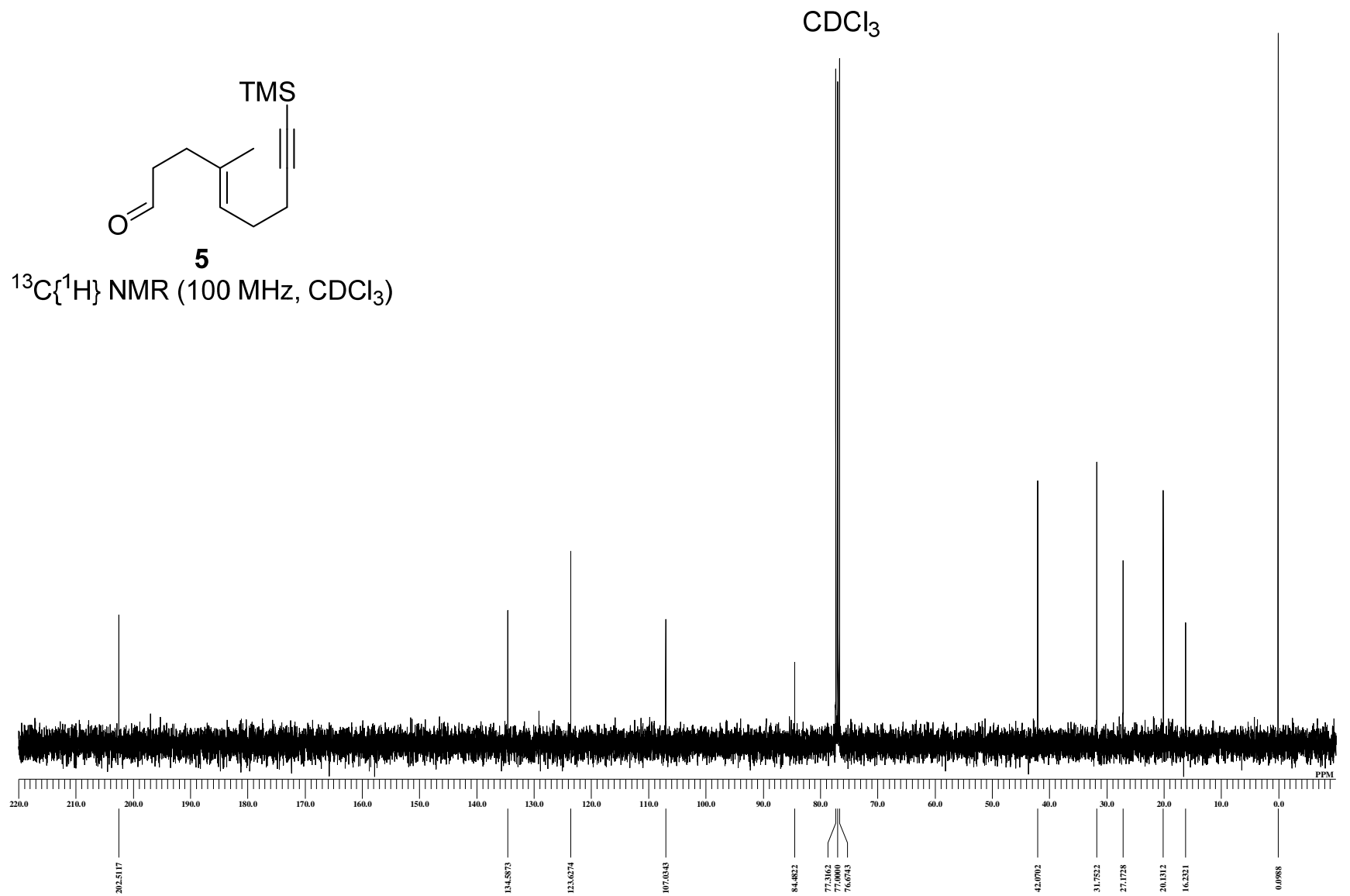




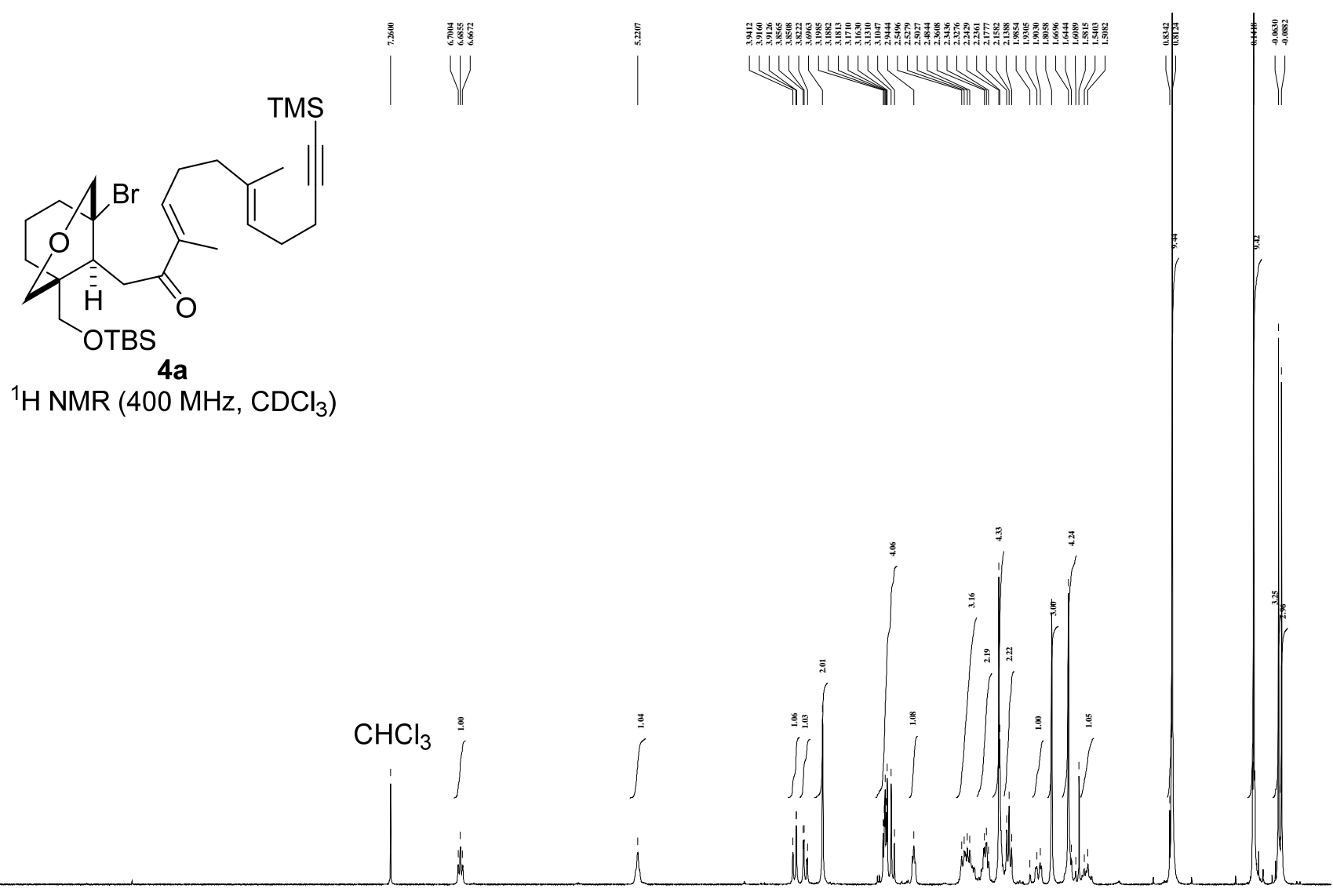

$\underset{1.5}{\prod_{10.5}}$

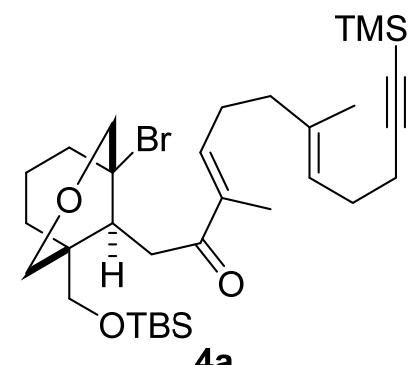

$\mathrm{CDCl}_{3}$

${ }^{13} \mathrm{C}\left\{{ }^{1} \mathrm{H}\right\} \mathrm{NMR}\left(100 \mathrm{MHz}, \mathrm{CDCl}_{3}\right)$

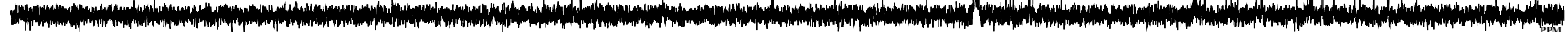
|

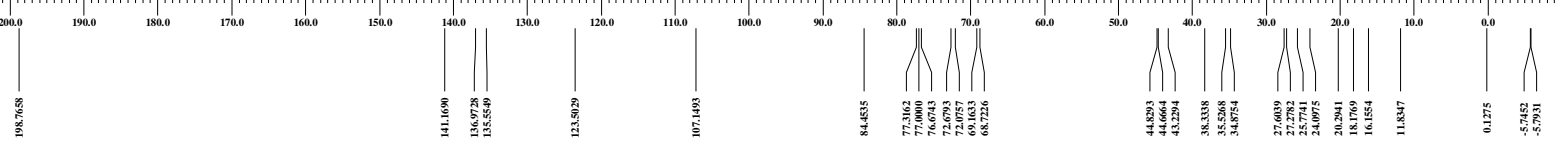




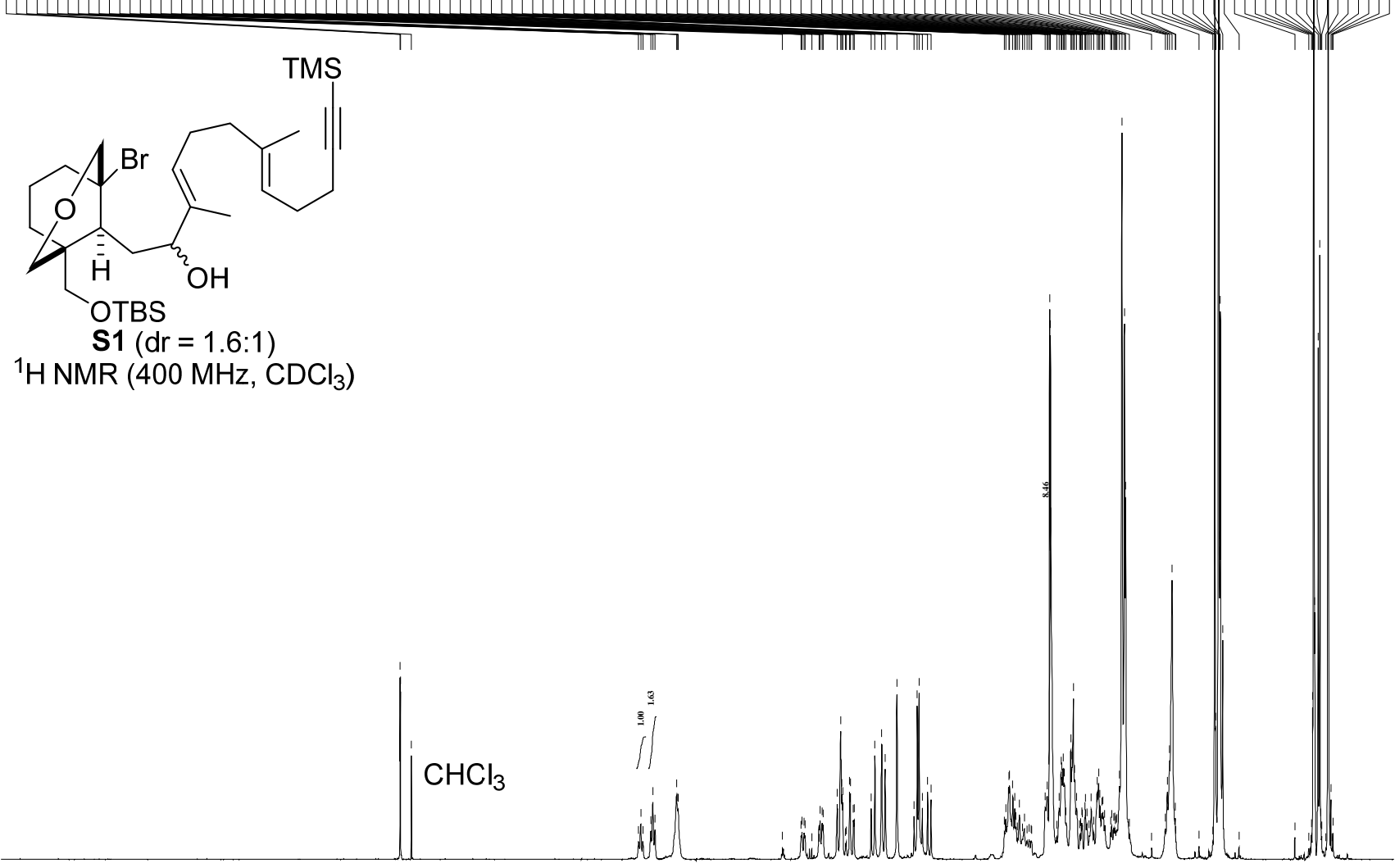

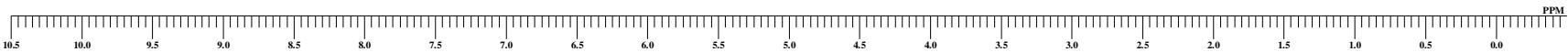




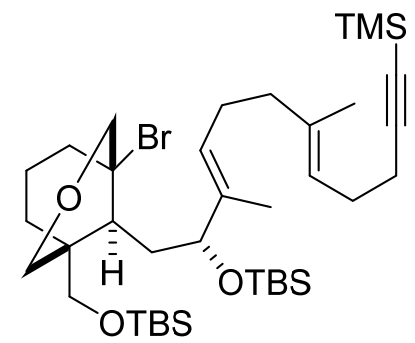

4b

${ }^{1} \mathrm{H} \mathrm{NMR}\left(400 \mathrm{MHz}, \mathrm{CDCl}_{3}\right)$

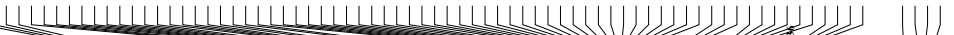

$\mathrm{CHCl}_{3}$

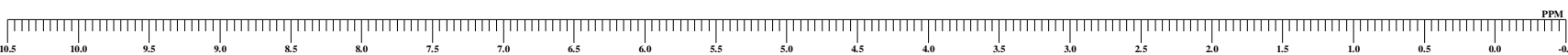

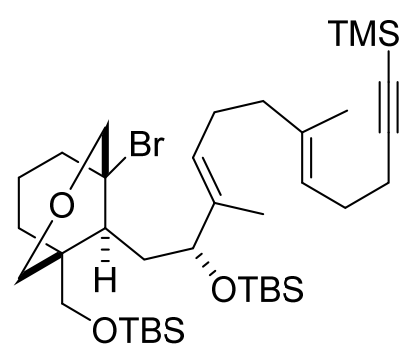

4b

${ }^{13} \mathrm{C}\left\{{ }^{1} \mathrm{H}\right\} \operatorname{NMR}\left(100 \mathrm{MHz}, \mathrm{CDCl}_{3}\right)$

$\mathrm{CDCl}_{3}$

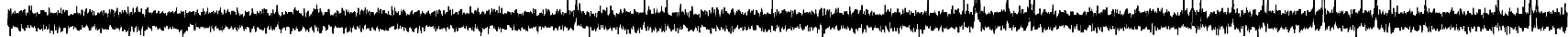

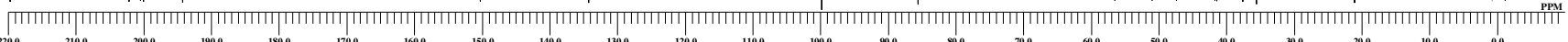

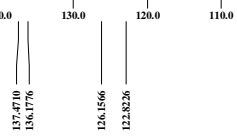

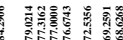

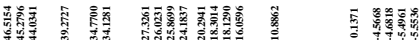




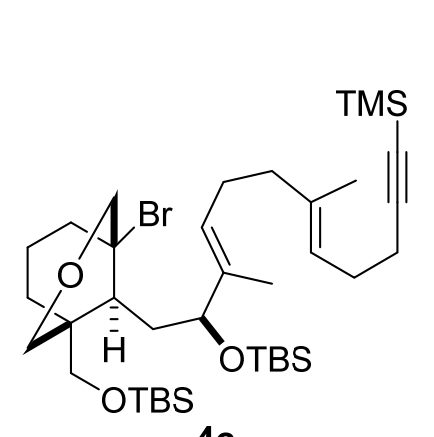

${ }^{1} \mathrm{H} \mathrm{NMR}\left(400 \mathrm{MHz}, \mathrm{CDCl}_{3}\right)$

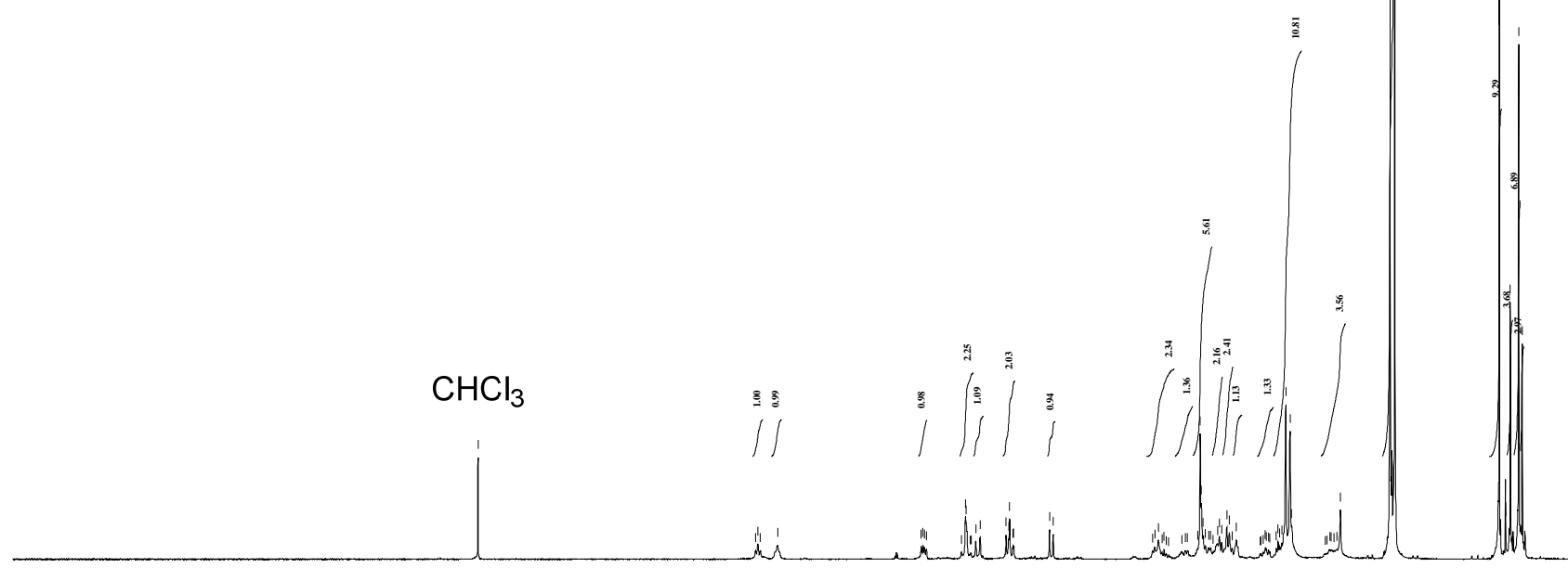

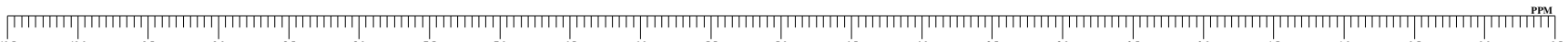

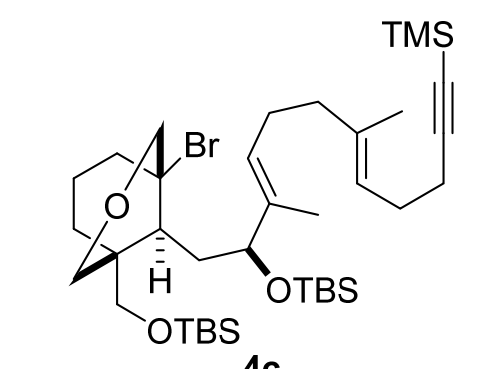

${ }^{13} \mathrm{C}\left\{{ }^{1} \mathrm{H}\right\} \mathrm{NMR}\left(100 \mathrm{MHz}, \mathrm{CDCl}_{3}\right)$

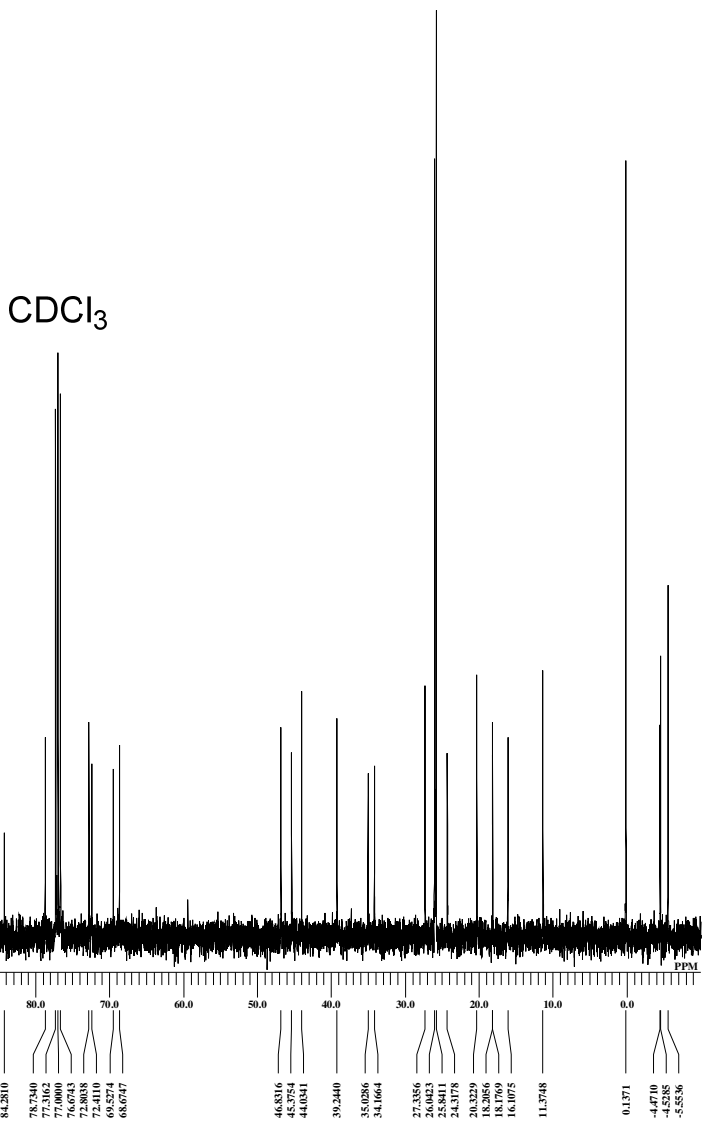



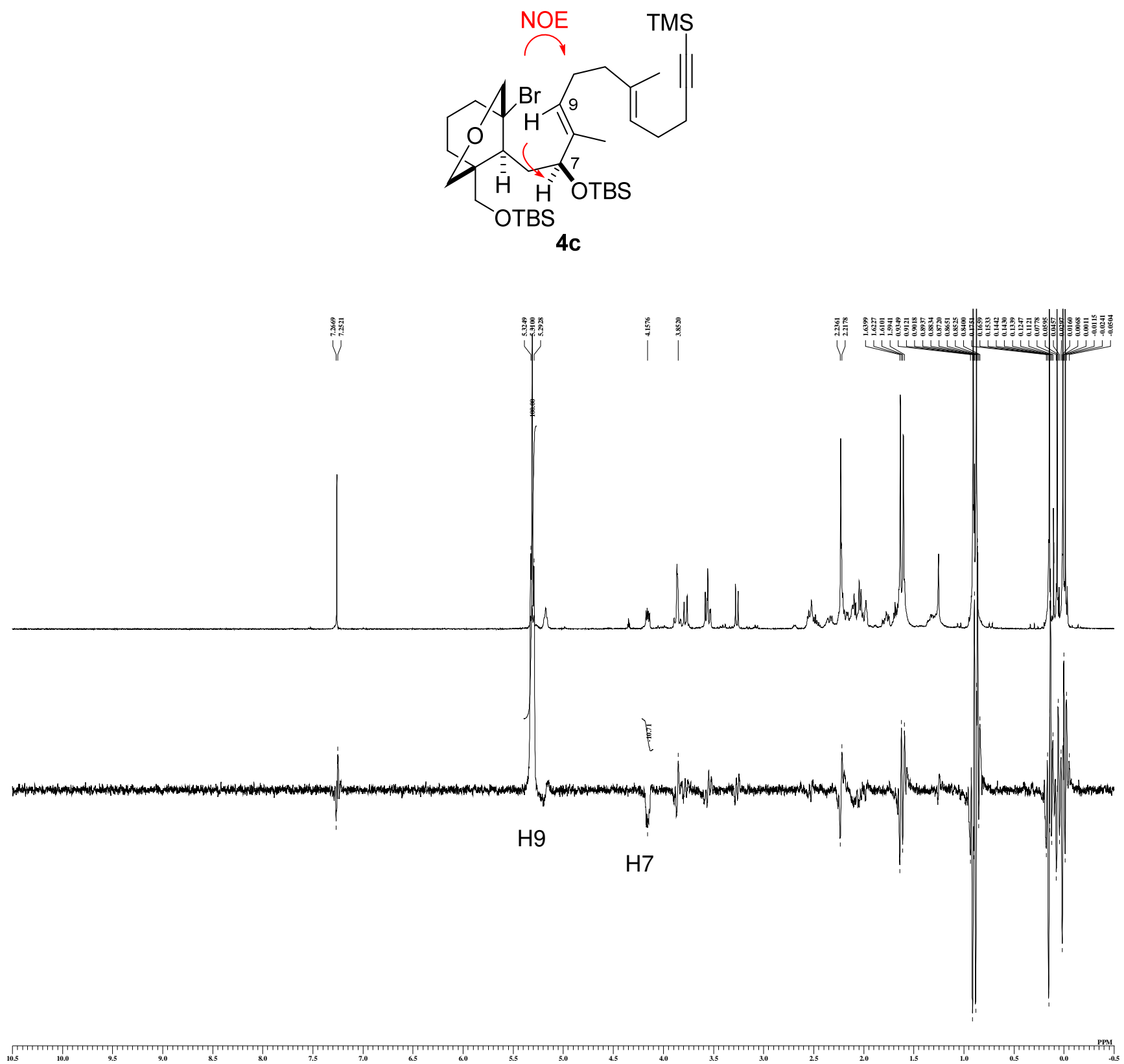


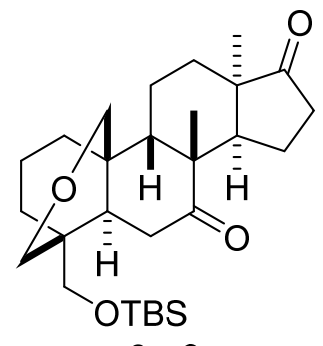

3a- $\beta$

${ }^{1} \mathrm{H}$ NMR $\left(400 \mathrm{MHz}, \mathrm{C}_{6} \mathrm{D}_{6}\right)$

$\mathrm{C}_{6} \mathrm{D}_{5} \mathrm{H}$

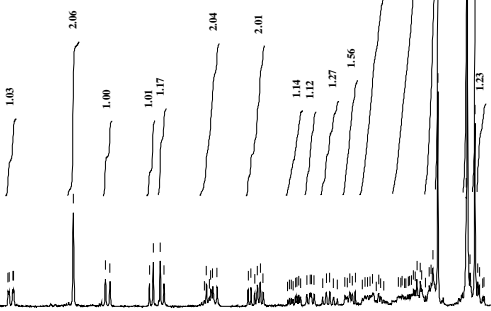

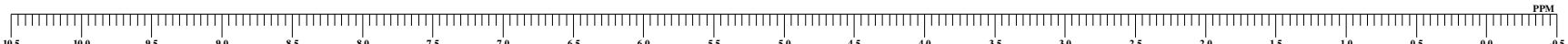

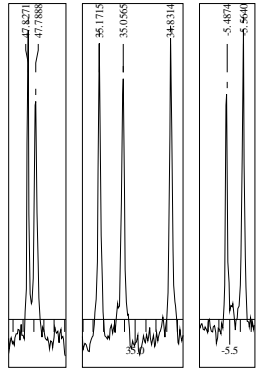

$\mathrm{C}_{6} \mathrm{D}_{6}$

${ }^{3} \mathrm{C}\left\{{ }^{1} \mathrm{H}\right\}$ NMR $\left(100 \mathrm{MHz}, \mathrm{C}_{6} \mathrm{D}_{6}\right)$ 

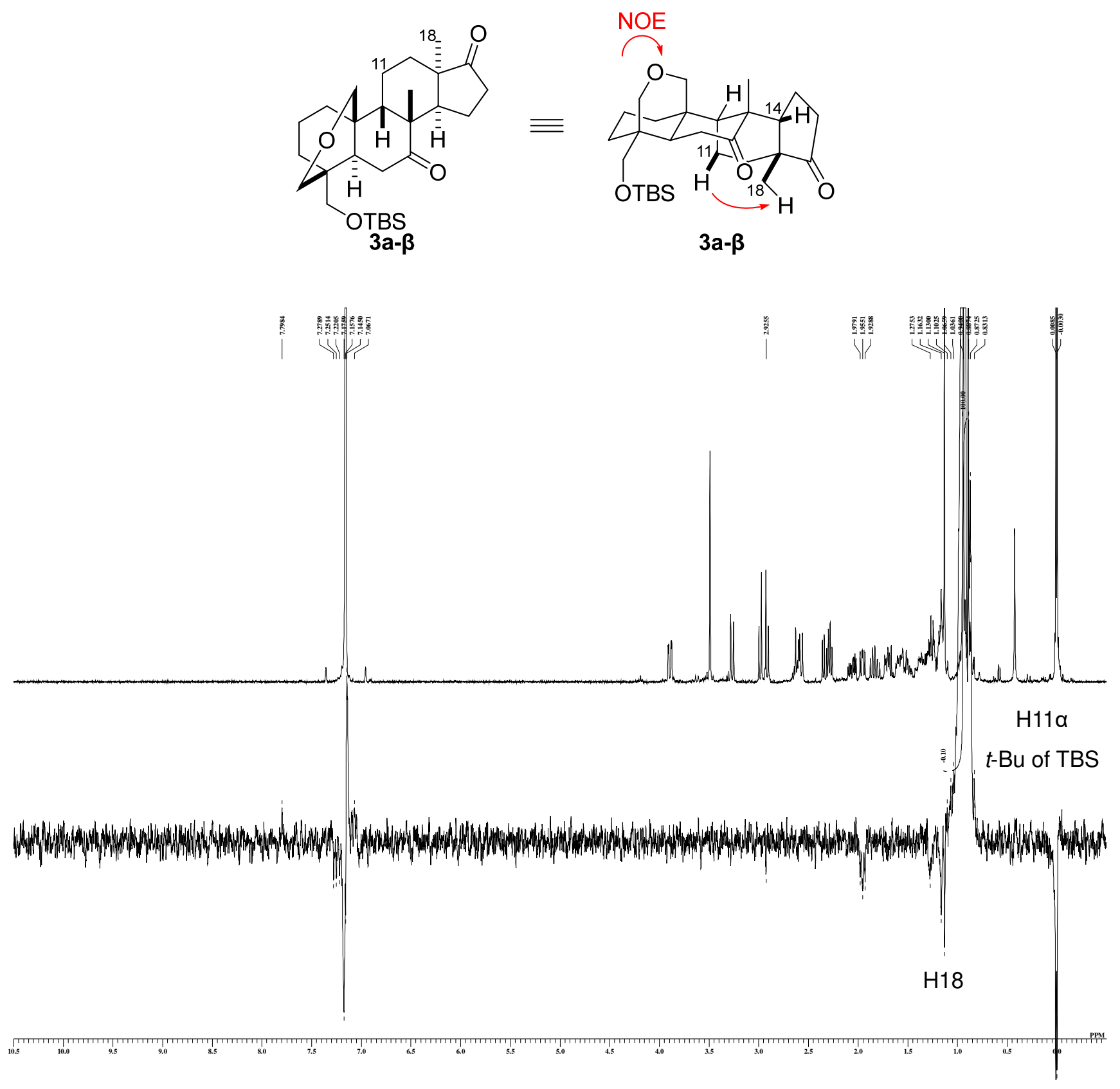

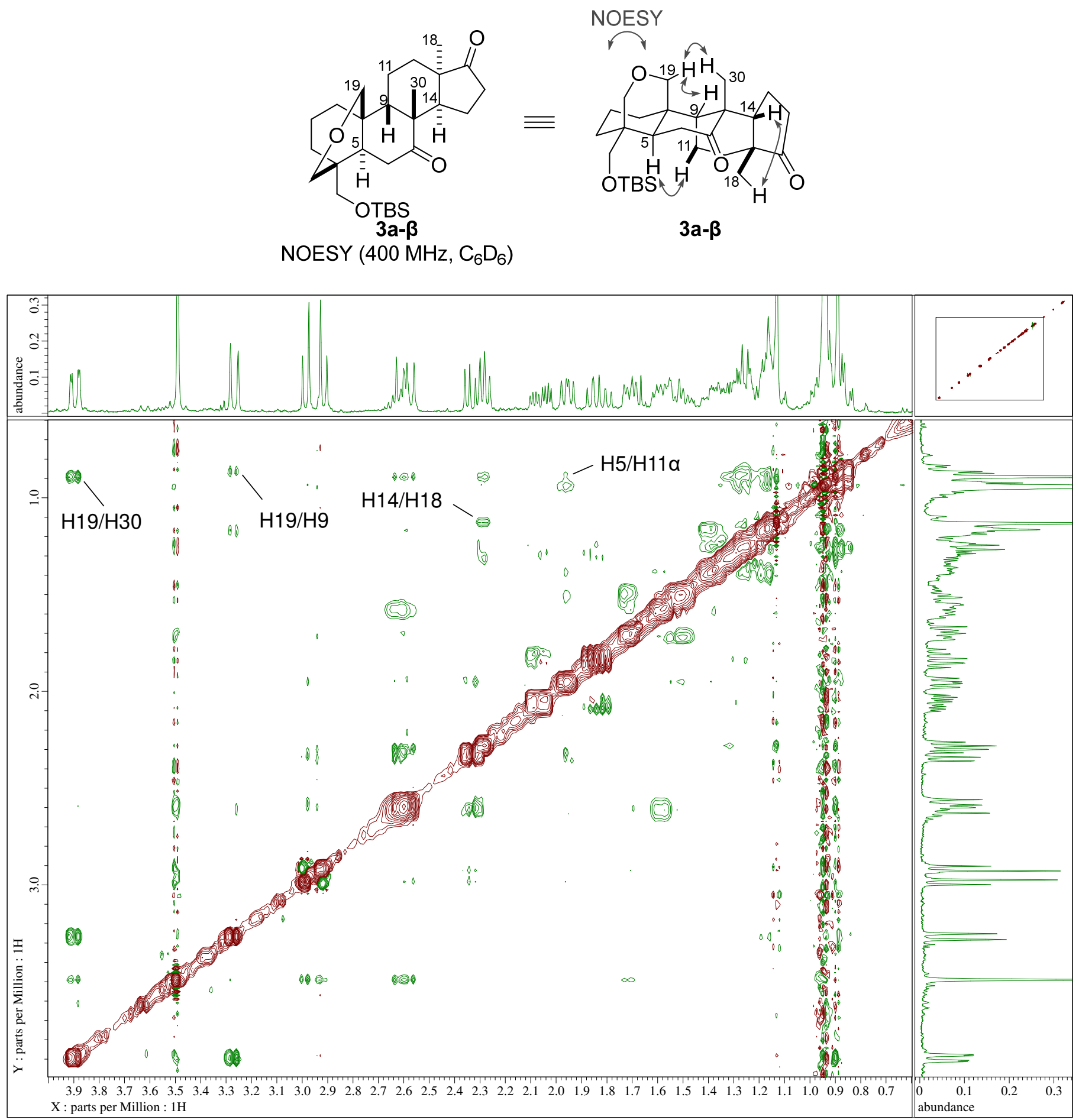


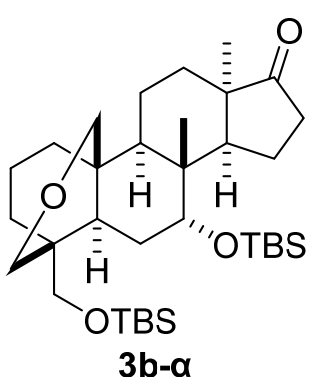

${ }^{1} \mathrm{H} \mathrm{NMR}\left(400 \mathrm{MHz}, \mathrm{CDCl}_{3}\right)$

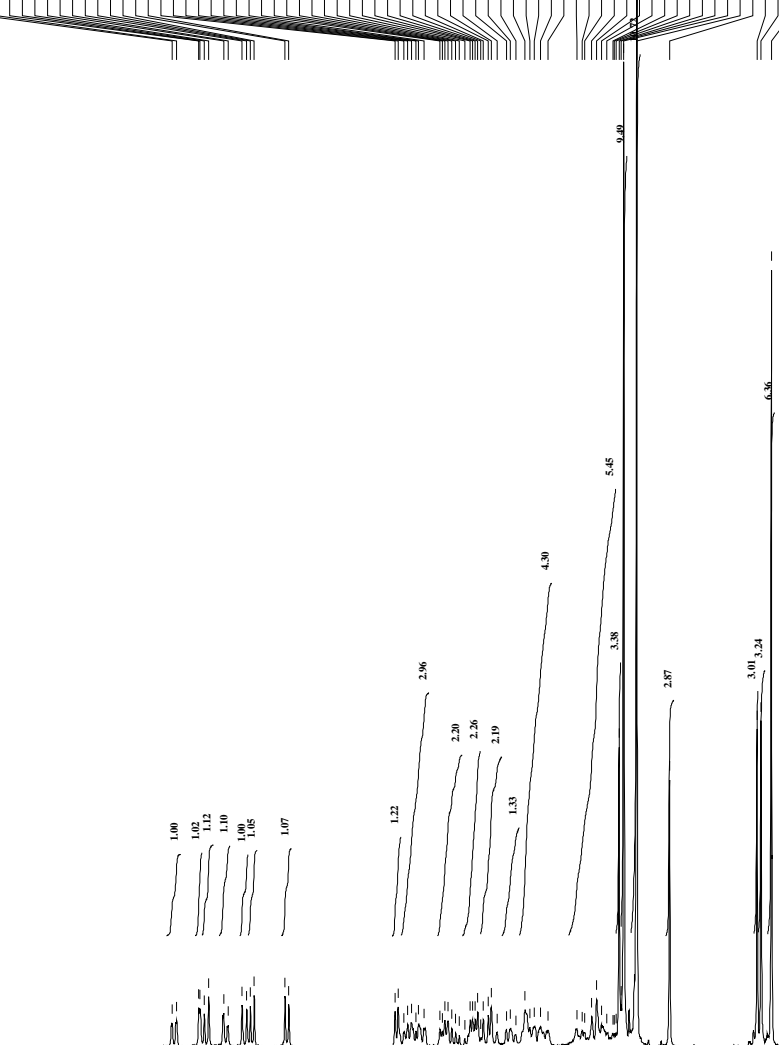

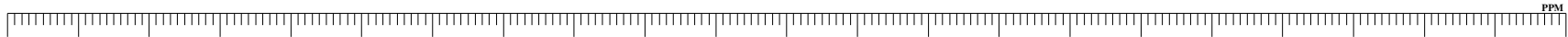
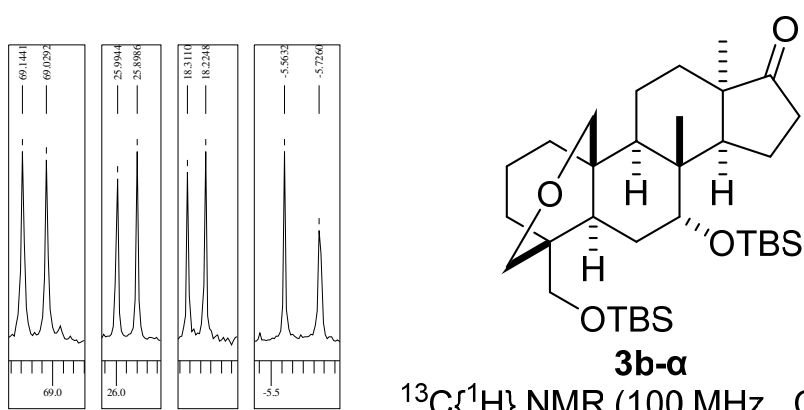

$\mathrm{CDCl}_{3}$

${ }^{13} \mathrm{C}\left\{{ }^{1} \mathrm{H}\right\} \operatorname{NMR}\left(100 \mathrm{MHz}, \mathrm{CDCl}_{3}\right)$

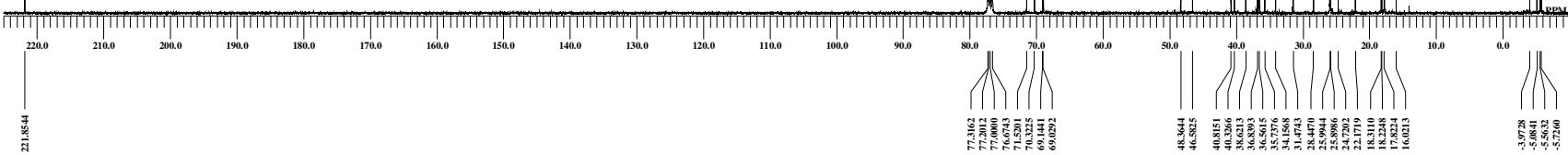



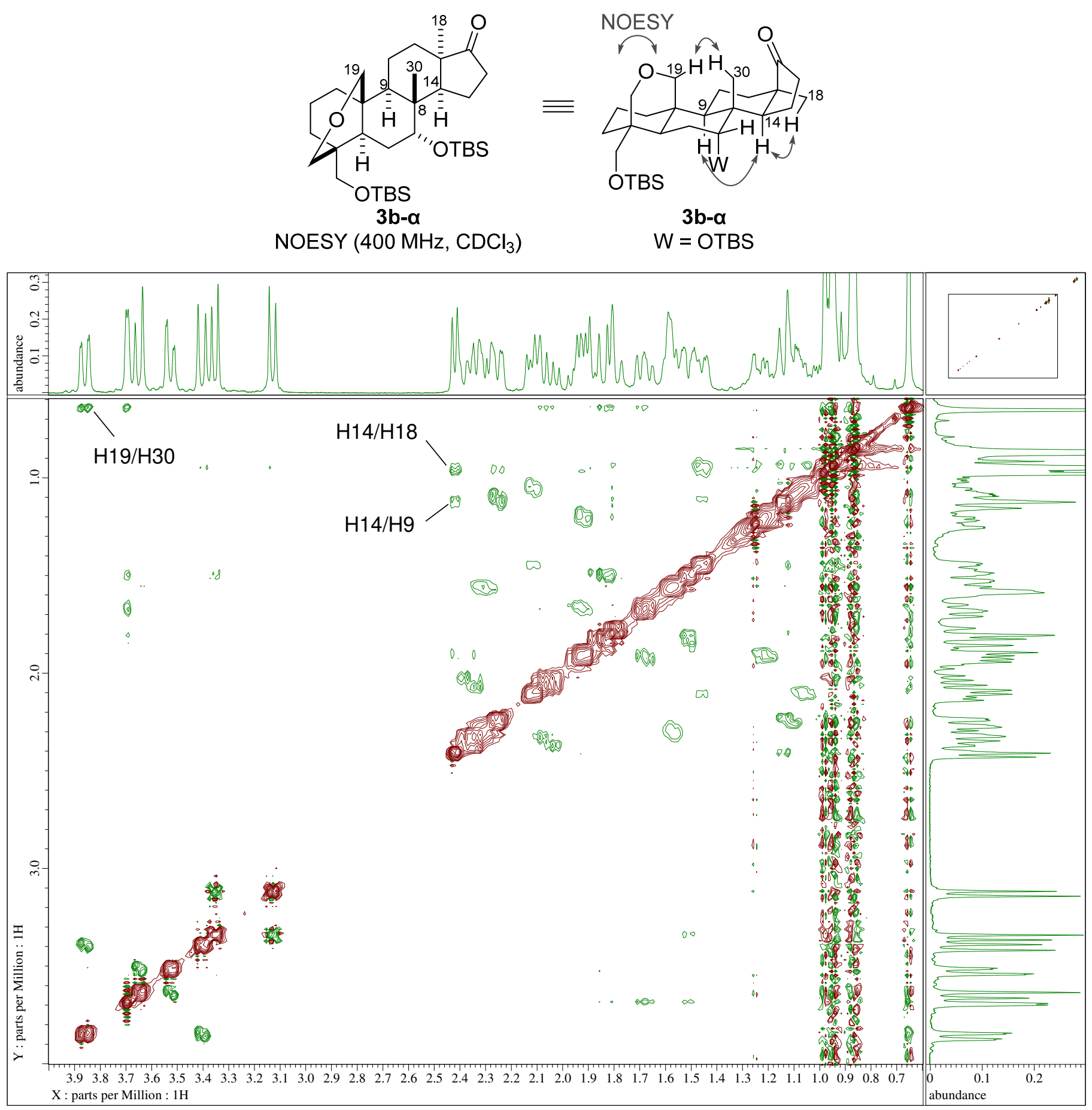


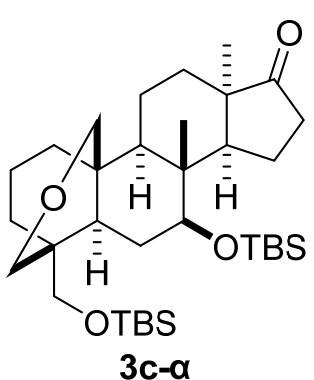

${ }^{1} \mathrm{H} \mathrm{NMR}\left(500 \mathrm{MHz}, \mathrm{CDCl}_{3}\right)$

$\mathrm{CHCl}_{3}$

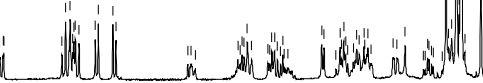

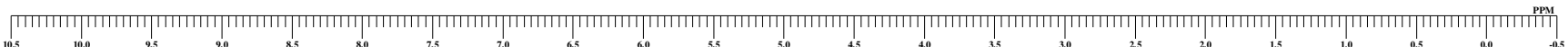
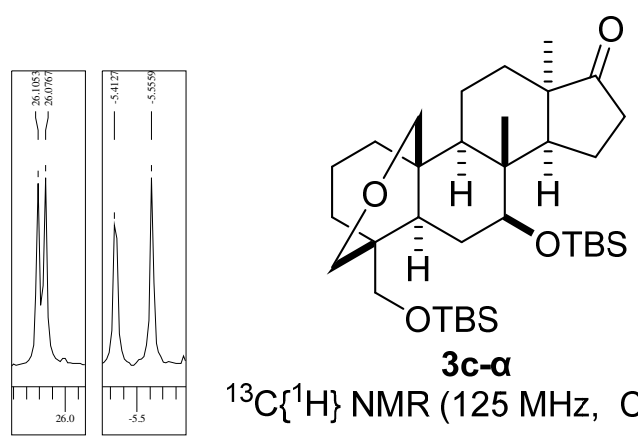

$\mathrm{CDCl}_{3}$

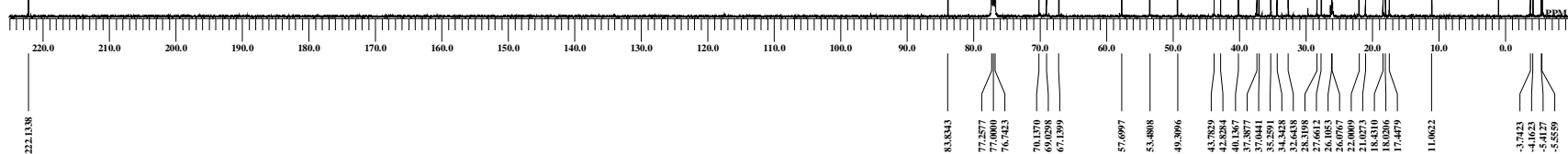




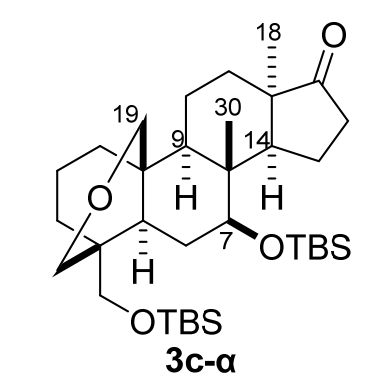

$\operatorname{NOESY}\left(500 \mathrm{MHz}, \mathrm{CDCl}_{3}\right)$
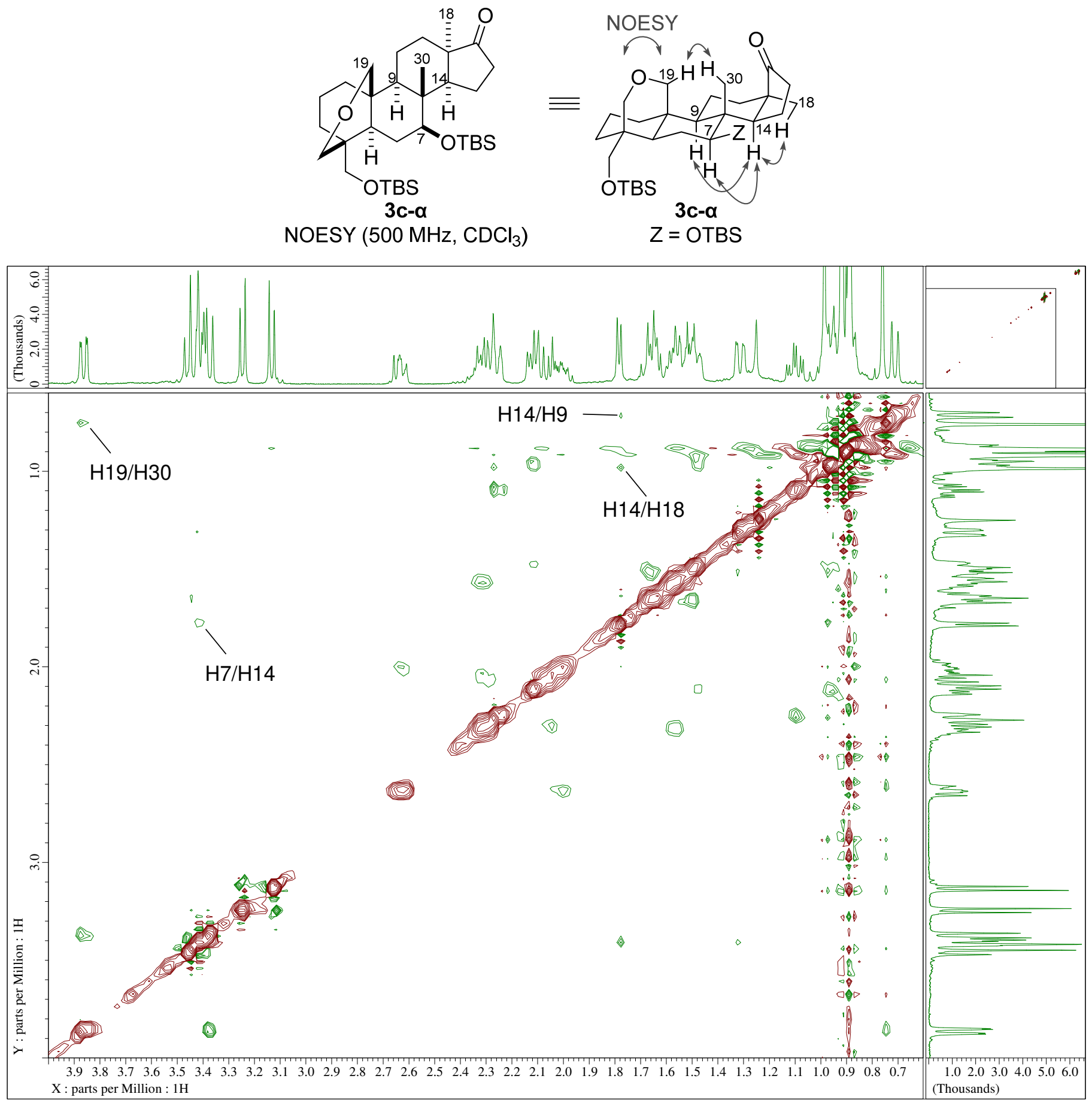